\title{
UNIFORM METRIC SPACES, ANNULAR QUASICONVEXITY AND POINTED TANGENT SPACES
}

\author{
DAVID A. HERRON* \\ (Dedicated to the memory of Juha Heinonen)
}

\begin{abstract}
We establish Väisälä's tangent space characterization for uniformity in the doubling metric space setting. We present similar results for other geometric properties.
\end{abstract}

\section{Introduction}

Uniform metric spaces were introduced in [5] and play a noteworthy role in the program of doing analysis in the metric space setting; these generalize the Euclidean uniform subspaces of $\mathbf{R}^{n}$ whose importance in geometric analysis is well established as documented in [12] and [27]. Euclidean uniform spaces were first studied by John [20] and Martio and Sarvas [25]. Every bounded Lipschitz domain in $\mathrm{R}^{n}$ is uniform, but a generic uniform space may have a fractal boundary.

Many important concepts in potential theory are known to hold in uniform spaces; for example, see [1] and [2]. There are close ties between uniformity and extension of Sobolev functions; see [21] for Euclidean space and [4] for the metric space setting. Recently, uniform subspaces of the Heisenberg groups, as well as more general Carnot groups, have become a focus of study; see [10], [9], [13].

Thanks to the aforementioned work of Bonk, Heinonen and Koskela, uniform metric spaces also feature prominently in geometric group theory. To wit, the quasihyperbolization of a (locally compact) uniform metric space is a (proper geodesic) Gromov hyperbolic space, and roughly speaking the converse holds as well.

The purpose of this article is to characterize the uniform subspaces of reasonable ambient spaces in term of tangent spaces. See Sections 2 and 3 for basic

\footnotetext{
* The author gratefully acknowledges support from the Charles Phelps Taft Research Center. Received 9 May 2009, in revised form 10 November 2009.
} 
information including definitions, notation and terminology; especially, $\S 3.2$ describes the families $\operatorname{Tan}(X)$ and $\operatorname{Tan}_{\mathrm{b}}(X, A)$ of pointed tangent spaces.

Roughly speaking, the uniformity of such a subspace $U$ is determined by the geometry of its boundary in the sense that $U$ is not uniform if and only if we can zoom in (or zoom out) at points of its boundary in such a way that either the boundary points look more and more like interior points of the complement, or the 'zoomed' complement of $U$ disconnects the 'zoomed' ambient space. The generic examples are $\mathrm{R} \times(0,1) \subset \mathrm{R}^{2}$ (zoom out at the origin) and $\mathrm{R}^{2} \backslash\{(x, 0): x \leq 1\}$ (zoom in at the origin).

TheOREM A. Let $X$ be a complete doubling annular quasiconvex metric space. Suppose $U$ is an open connected subspace of $X$ with $\mathrm{bd}(U) \neq \emptyset$. Put $A:=X \backslash U$. Then $U$ is uniform if and only if for each $\left(X_{\infty}, A_{\infty} ; a_{\infty}\right)$ in $\operatorname{Tan}_{\mathrm{b}}(X, A)$, the point $a_{\infty}$ belongs to $\mathrm{bd}\left(A_{\infty}\right)$ and $X_{\infty} \backslash A_{\infty}$ is connected.

Our proof relies on a similar characterization for plump open subspaces. In the length space setting, these are the subspaces $U$ that have the property that for each metric ball $B$ centered in $U$ there is another comparably sized metric ball that is centered in $B$ and contained in $U$. An infinite cylinder in Euclidean space does not possess this property.

Proposition B. Let $X$ be a complete doubling length metric space. Suppose $U$ is an open subspace of $X$ with $\operatorname{bd}(U) \neq \emptyset$. Put $A:=X \backslash U$. Then $U$ is plump in $X$ if and only if for each $\left(X_{\infty}, A_{\infty} ; a_{\infty}\right)$ in $\operatorname{Tan}_{\mathrm{b}}(X, A), a_{\infty} \in \operatorname{bd}\left(A_{\infty}\right)$.

In addition, we utilize the following plumpness characterization for uniformity.

Proposition C. Let $U$ be a non-complete locally complete metric space. Suppose $U$ is c-plump and 3 c-proximate points in $U$ can be joined by b-uniform paths. Then $U$ is a-uniform with $a=18 b^{2} c$. The converse holds with $c=4 a$ and $b=a$.

Note that in contrast to the above, neither Theorem A nor Proposition B is quantitative. In the Euclidean space setting, these three results are due to Väisälä; see [27, Theorems 2.15, 3.5, 3.8].

A natural question to ask is what other geometric properties of spaces can be characterized in terms of their tangent spaces. As described in Proposition 2.3, there is a close connection between annular quasiconvexity and uniformity, so the following is not surprising.

Theorem D. Let $X$ be a complete doubling length metric space. Then $X$ is annular quasiconvex if and only if for each $\left(X_{\infty} ; a_{\infty}\right)$ in $\operatorname{Tan}(X), X_{\infty} \backslash\left\{a_{\infty}\right\}$ is connected. 
Our final result suggests that the bounded turning and linear local connectivity properties cannot be detected at the tangent space level.

Example E. Let $C \subset \mathrm{R}^{n}$ be closed and $a \in C$. There exists a 1-bounded turning 1-linearly locally connected open connected $U \subset \mathrm{R}^{n}$ with $(C ; a)$ in $\operatorname{Tan}\left(\mathrm{R}^{n} \backslash U\right)$.

The necessary conditions in each of Theorems A and D and Proposition B above can be strengthened as indicated in Section 4. Roughly speaking, the geometric property of interest is always inherited by the tangent space.

This document is organized as follows: Section 2 contains preliminary information including basic definitions and terminology descriptions; e.g., our definition of uniform spaces is given in \$2.2.2. Section 3 includes a discussion of pointed Gromov-Hausdorff distance as well as the construction for Example E. We establish the above results in Section 4.

The author is grateful to Stephen Buckley and Nageswari Shanmugalingam for helpful discussions. The author thanks the referee for their insightful suggestions. After preparing this manuscript, the author learned that Xiangdong Xie has also proven Theorem D.

\section{Preliminaries}

Here we set forth our (relatively standard) notation and terminology and provide fundamental definitions and basic information. For real numbers $a$ and $b$,

$$
a \wedge b:=\min \{a, b\} \quad \text { and } \quad a \vee b:=\max \{a, b\} .
$$

\subsection{Metric Space Definitions}

Throughout this article $(X, d)$ denotes a general metric space which we often refer to as just $X$. In this setting, all topological notions refer to the metric topology; here $\mathrm{cl}(A), \operatorname{bd}(A), \operatorname{int}(A)$ are the closure, boundary, interior (respectively) of $A \subset X$. We write $\bar{X}=\bar{X}_{d}$ and $\partial X=\partial_{d} X:=\bar{X}_{d} \backslash X$ to denote the metric completion and metric boundary, respectively, of $(X, d)$. We note that when $A$ is an open subspace of $X, \operatorname{bd}(A) \subset \partial A$ and equality holds if $X$ is complete (but not in general). When there are several metric spaces under consideration, such as $X$ and $Y$, we denote the distance functions as $d_{X}$ and $d_{Y}$, respectively, if there is any chance of confusion.

In general, we write the distance between points $x, y$ as $|x-y|=d(x, y)$. The open and closed balls of radius $r$ centered at the point $x$ are $\mathrm{B}(x ; r):=$ $\mathrm{B}_{d}(x ; r):=\{y:|x-y|<r\}$ and $\overline{\mathrm{B}}(x ; r):=\{y:|x-y| \leq r\}$. The closed annular ring centered at $x$ with inner radius $r$ and outer radius $s$ is

$$
\mathrm{A}(x ; r, s):=\overline{\mathrm{B}}(x ; s) \backslash \mathrm{B}(x ; r)=\{y: r \leq|x-y| \leq s\} .
$$


The open $t$-neighborhood about $A \subset X$ is

$$
\mathrm{N}[A ; t]:=\{x \in X \mid \operatorname{dist}(x, A)<t\}=\bigcup_{a \in A} \mathrm{~B}(a ; t) .
$$

For convenience, we set

$$
\Delta(X):= \begin{cases}(0, \operatorname{diam}(X)] & \text { when } X \text { is bounded } \\ (0, \infty) & \text { otherwise }\end{cases}
$$

A metric space $X$ is locally complete provided $d(x):=\operatorname{dist}(x, \partial X)>0$ for every $x \in X$; equivalently, $X$ is an open subspace of $\bar{X}$. In a non-complete locally complete space $X$ we write

$$
\mathrm{B}(x):=\mathrm{B}(x ; d(x)) \quad \text { and for any } c>0, \quad c \mathrm{~B}(x):=\mathrm{B}(x ; c d(x)) .
$$

Two points $x, y$ are a-proximate, for some constant $a>0$, if $|x-y| \leq$ $a[d(x) \wedge d(y)]$. If this holds, then also $(a+1)^{-1} \leq d(x) / d(y) \leq a+1$.

A metric space $X$ is doubling, or, satisfies a (metric) doubling condition if there is a constant $v$ such that each ball in $X$ of radius $r$ can be covered by using at most $v$ balls of radius $r / 2$; these are precisely the spaces of finite Assouad dimension. In other words, for all $x \in X$ and all $r>0, N(r ; \mathrm{B}(x ; 2 r)) \leq v$, where

$$
N(r ; E):=\min \left\{n \in \mathrm{N} \mid \exists x_{1}, \ldots, x_{n} \text { st } E \subset \cup_{i=1}^{n} \mathrm{~B}\left(x_{i} ; r\right)\right\} .
$$

Examples of doubling spaces include all Euclidean spaces, Heisenberg groups, and Ahlfors regular spaces.

Note that balls in doubling spaces are totally bounded. Thus every complete doubling space is proper (i.e., has the Heine-Borel property that closed bounded subsets are compact).

A path is a continuous map of an interval; all path parametrization intervals are assumed to be compact unless explicitly indicated otherwise. The notation $|\gamma|$ stands for the trajectory (i.e., image) of a path $\gamma$. For a path $\gamma$, the phrase $\gamma$ joins $x$ to $y$ is also meant to describe an orientation, and when $\gamma$ is injective, we write $\gamma[x, y], \gamma(x, y), \gamma[x, y)$ for the various (closed, open, etc.) subpaths of $\gamma$ that join $x$ to $y$. We also use this notation for a general path $\gamma$; here $\gamma[x, y]$ denotes the unique injective subpath of $\gamma$ that joins $x, y$ obtained by using the last time $\gamma$ is at $x$ up to the first time $\gamma$ is at $y$.

When $\alpha$ and $\beta$ are paths that join $x$ to $y$ and $y$ to $z$ respectively, we write $\alpha \star \beta$ for the concatenation of $\alpha$ and $\beta$; so $\alpha \star \beta$ joins $x$ to $z$. Of course, $|\alpha \star \beta|=|\alpha| \cup|\beta|$. 
We mention the useful fact that every path contains an injective subpath that joins its endpoints. This observation follows by cutting out loops; see [28].

The length of a path $[0,1] \stackrel{\gamma}{\rightarrow} X$ is defined by

$$
\ell(\gamma):=\sup \left\{\sum_{i=1}^{n}\left|\gamma\left(t_{i}\right)-\gamma\left(t_{i-1}\right)\right|: 0=t_{0}<t_{1}<\cdots<t_{n}=1\right\},
$$

and $\gamma$ is rectifiable when $\ell(\gamma)<\infty$. A geodesic in $X$ is the trajectory (image) $|\gamma|$ of some isometric embedding $I \stackrel{\gamma}{\rightarrow} X$ where $I \subset \mathrm{R}$ is an interval; we use the adjectives segment, ray, or line (respectively) to indicate that $I$ is bounded, semi-infinite, or all of R.

A metric space is geodesic if each pair of points can be joined by a geodesic segment. We use the notation $[x, y]$ to mean a (not necessarily unique) geodesic segment joining points $x, y$; such geodesics always exist if our space is geodesic, but may not be unique. We consider a given geodesic $[x, y]$ as being ordered from $x$ to $y$ (so we can use phrases such as the 'first' point encountered). Abusing notation, we also view a given $[x, y]$ as the path $[0,|x-y|] \ni t \mapsto$ $\gamma(t) \in[x, y]$ where $|\gamma(t)-x|=t$; this permits us to write expressions such as $[x, y] \star[y, z]$.

\subsection{Annular Quasiconvex, Uniform, and Plump Spaces}

A rectifiable path $\gamma$ with endpoints $x, y$ is a $c$-quasiconvex path, $c \geq 1$ some constant, provided $\ell(\gamma) \leq c|x-y|$. A metric space is $c$-quasiconvex if each pair of points in it can be joined by a $c$-quasiconvex path. (Note that in general the trajectory of a quasiconvex path need not be quasiconvex.) Thus a metric space is: quasiconvex if and only if it is bilipschitz equivalent to a length space, a length space if and only if it is $c$-quasiconvex for each $c>1$, and a geodesic space if and only if it is 1-quasiconvex.

2.2.1. Annular QuasiConvexity. A metric space $X$ is $c$-annular quasiconvex at $p \in X, c \geq 1$, provided it is connected and for all $r>0$, points in $\mathrm{A}(p ; r, 2 r)$ can be joined by $c$-quasiconvex paths lying in $\mathrm{A}(p ; r / c, 2 c r)$. We call $X c$ annular quasiconvex if it is $c$-annular quasiconvex at each point. Examples of quasiconvex and annular quasiconvex metric spaces include Banach spaces and upper regular Loewner spaces; the latter includes Carnot groups and certain Riemannian manifolds with non-negative Ricci curvature; see [16, 3.13, 3.18, Section 6]. Korte [22] has recently verified that doubling metric measure spaces that support a $(1, p)$-Poincaré inequality with sufficiently small $p$ are annular quasiconvex.

To the best of our knowledge, the notion of annular quasiconvexity was first introduced in [22] and [7]; it was an essential ingredient in [19]. A similar concept has recently been employed in [23]. 
The assumption that an annular quasiconvex space is connected can be relaxed, e.g., to something like uniformly perfect. We want to rule out spaces such as $\{0\} \cup[1,2] \cup[100,101] \cup[10000,10002] \ldots$. We also note that any $c$-quasiconvex path joining points in a ball $\overline{\mathrm{B}}(p ; r)$ will lie in $\overline{\mathrm{B}}(p ;(c+1) r)$. To see this, assume $z \in|\gamma|$ for some such path $\gamma$ joining $x, y \in \overline{\mathrm{B}}(p ; r)$ and note that

$$
|z-p| \leq(|z-x|+|x-p|) \wedge(|z-y|+|y-p|) \leq r+\ell(\gamma) / 2 \leq(c+1) r
$$

The important consequence of annular quasiconvexity, versus quasiconvexity, is that we can join points by avoiding the centers of such balls.

Here are some elementary properties of annular quasiconvex spaces. For example, with regards to annular quasiconvexity, there is no harm in assuming that our space is complete. See [7, Propositions 6.1, 6.3] and [18, Theorem 2.7] for ideas behind the proofs of the following.

FACT 2.2. Let $X$ be annular $c$-quasiconvex at some point $p$. Then:

(a) $X$ is $9 c$-quasiconvex.

(b) $\bar{X}$ is $10 c$-annular quasiconvex at $p$.

(c) points in $\mathrm{A}(p ; r, R)$ can be joined in $\mathrm{A}(p ; r / c, c R)$ by $45 c$-quasiconvex paths.

There is an intimate connection between the annular quasiconvexity of a space and uniformity of certain of its subspaces; the definition of a uniform space is given in §2.2.2. For related information see [7, §6.C]. Roughly speaking, annular quasiconvex spaces are those for which single points are removable for uniformity.

A rectifiable path $\gamma$ with endpoints $x, y$ is a $c$-cone path from $x$ to $y$ provided $c \geq 1$ and

$$
\forall z \in|\gamma|, \quad \ell(\gamma[x, z]) \leq c d(z) ;
$$

here we assume that $x, y, \gamma$ lie in some non-complete locally complete space $U$ and $d(z):=\operatorname{dist}(z, \partial U)$.

Proposition 2.3. Let $X$ be a complete connected metric space. Fix $p \in X$. The following are quantitatively equivalent:

(a) $X$ is c-annular quasiconvex at $p \in X$.

(b) $\forall x, y \in X \backslash\{p\}$ satisfying $|x-p| \leq|y-p|, \exists$ a c-quasiconvex c-cone path from $x$ to $y$.

(c) $X \backslash\{p\}$ is c-uniform.

The constant c varies from (a) to (b) to (c), but each depends only on the other. 
Proof. We establish (a) $\Rightarrow(\mathrm{b}) \Rightarrow(\mathrm{c}) \Rightarrow(\mathrm{a})$. The middle implication is trivial. To prove (c) $\Rightarrow$ (a), assume $X \backslash\{p\}$ is $c$-uniform and fix points $x, y$ in some annular ring $\mathrm{A}(p ; r, 2 r)$. Let $\gamma$ be a $c$-uniform path joining $x, y$. Then as in (2.1), $|\gamma| \subset \overline{\mathrm{B}}(p ; 2(c+1) r)$. Also for each $z \in|\gamma|$ : if $\operatorname{dist}(z,\{x, y\}) \leq r / 2$, then $|z-p| \geq r / 2$ whereas when $\operatorname{dist}(z,\{x, y\}) \geq r / 2$,

$$
|z-p|=d(z) \geq c^{-1}[\ell(\gamma[x, z]) \wedge \ell(\gamma[y, z])] \geq r /(2 c) .
$$

Thus in all cases $|\gamma| \subset \mathrm{A}(p ; r / 2 c, 2(c+1) r)$; so, $X$ is $2(c+1)$-annular quasiconvex at $p$.

To prove (a) $\Rightarrow(\mathrm{b})$, assume $X$ is $c$-annular quasiconvex at $p$. Fix points $x, y \in X \backslash\{p\}$ with $r:=|x-p| \leq|y-p|$. Suppose first that $|y-p| \leq 2 r$. There is a $c$-quasiconvex path $\gamma$ joining $x, y$ in $\mathrm{A}(p ; r / c, 2 c r)$. Then $\ell(\gamma) \leq$ $3 c r$, so for all $z \in|\gamma|$,

$$
d(z)=|z-p| \geq \frac{r}{c} \geq \frac{\ell(\gamma)}{3 c^{2}}
$$

and thus $\gamma$ is a $3 c^{2}$-cone path from $x$ to $y$.

Now suppose $|y-p|>2 r$ and pick $n \in \mathrm{N}$ with $2^{n} r<|y-p| \leq 2^{n+1} r$. Put $x_{0}:=x, x_{n+1}:=y$ and for each $1 \leq i \leq n$ select a point $x_{i}$ with $\left|x_{i}-p\right|=r_{i}:=2^{i} r$. For each $1 \leq i \leq n+1$, there are $c$-quasiconvex paths $\alpha_{i}$ joining $x_{i-1}, x_{i}$ in $\mathrm{A}\left(p ; r_{i-1} / c, c r_{i}\right)$. We claim that $\gamma:=\alpha_{1} \star \cdots \star \alpha_{n+1}$ is a $b$-quasiconvex $b$-cone path from $x$ to $y$ with $b:=3 c(3 \wedge 2 c)$. To check the $b$-quasiconvex property, we calculate

$$
\begin{aligned}
\ell(\gamma) & =\sum_{i=1}^{n+1} \ell\left(\alpha_{i}\right) \leq c \sum_{i=1}^{n+1}\left|x_{i}-x_{i-1}\right| \leq 3 c r \sum_{i=1}^{n+1} 2^{i-1} \\
& =3 c\left(2^{n+1}-1\right) r<3 c \frac{2^{n+1}-1}{2^{n}-1}|x-y| \leq 9 c|x-y| .
\end{aligned}
$$

The penultimate inequality above holds because $|x-y| \geq|y-p|-|x-p| \geq$ $2^{n} r-r$.

It remains to verify the $b$-cone property. Let $z \in|\gamma|$, say $z \in\left|\alpha_{j}\right|$ for some $1 \leq j \leq n+1$. The choice of $\alpha_{j}$ ensures that

$$
d(z)=|z-p| \geq r_{j-1} / c=2^{j-1} r / c .
$$

Thus

$$
\ell(\gamma[z, x]) \leq \sum_{i=1}^{j} \ell\left(\alpha_{j}\right) \leq c \sum_{i=1}^{j}\left|x_{i}-x_{i-1}\right| \leq 3 c r \sum_{i=1}^{j} 2^{i-1} \leq 6 c^{2} d(z) .
$$


2.2.2. Uniformity. Roughly speaking, a space is uniform when points in it can be joined by paths that are not too long and that move away from the boundary. A non-complete locally complete metric space $U$ is a uniform space if there is a constant $c \geq 1$ such that each pair of points in $U$ can be joined by a $c$-uniform path. A rectifiable path $\gamma$ that joins points $x, y$ in such a metric space $U$ is a $c$-uniform path provided it is both $c$-quasiconvex and

$$
\forall z \in|\gamma|, \quad \ell(\gamma[x, z]) \wedge \ell(\gamma[y, z]) \leq c d(z),
$$

where $d(z):=\operatorname{dist}(z, \partial U)$. We call $\gamma$ a double $c$-cone path if it satisfies the above condition (the phrases cigar path and corkscrew are also used). In [27] Väisälä provides a description of various possible double cone conditions (which he calls length cigars, diameter cigars, distance cigars, and Möbius cigars). Martio's work [24] should also be mentioned.

An elementary, but useful, observation is that any path $\gamma$ in $U$ with endpoints $x, y$ is a $c$-uniform path where $c:=\ell(\gamma) /[|x-y| \wedge \operatorname{dist}(|\gamma|, \partial U)]$. Here are some additional facts.

LEMMA 2.4. Let $U \subset X$ be an open subspace of a complete geodesic space $X$ with $\operatorname{bd}(U) \neq \emptyset$. Suppose $x, y \in U$ with $d(x) \leq d(y)$. If $y \in \overline{\mathrm{B}}(x)$, then every geodesic $[x, y]$ is a 1-uniform path in $U$.

Proof. Select $w \in[x, y]$ with $|x-w|=|x-y| / 2=|y-w|$. Suppose $z \in[x, w]$. Then $|x-z| \leq|x-w|$, so

$$
d(z) \geq d(x)-|x-z| \geq d(x)-\frac{|x-y|}{2} \geq \frac{|x-y|}{2} \geq|x-z|=\ell([x, z]) .
$$

Since $d(y) \geq d(x), x \in \overline{\mathrm{B}}(y)$ and so the same argument applies for $z \in[y, w]$.

LemMA 2.5. Let $U \subset X$ be an open subspace of a complete geodesic $b$ annular quasiconvex space $X$ with $\operatorname{bd}(U) \neq \emptyset$. Fix $a \geq 1+2 b$ and $x, y \in U$ with $d(x) \leq d(y)$. Pick $u \in \operatorname{bd}(U)$ with $r:=d(x)=|x-u|$. Suppose

$$
r \leq|x-y| \leq a r \quad \text { and } \quad A:=(X \backslash U) \cap \overline{\mathrm{B}}(x ; 3 \text { ar }) \subset \overline{\mathrm{B}}(u ; r / 2 b) .
$$

Then there is a c-uniform path in $U$ joining $x$ and $y$, where $c:=b(a \vee 6 b)$.

Proof. Note that $y \notin \mathrm{B}(u ; r)$, and for all $z \in a \overline{\mathrm{B}}(x), d(z):=\operatorname{dist}(z, \operatorname{bd}(U))$ $=\operatorname{dist}(z, A)$.

Suppose $y \in \overline{\mathrm{B}}(u ; 2 r)$. Then $x, y \in \mathrm{A}(u ; r, 2 r)$. Since $X$ is $b$-annular quasiconvex, there is a $b$-quasiconvex path $\gamma$ joining $x, y$ in $\mathrm{A}(u ; r / b, 2 b r)$. In particular, $\ell(\gamma) \leq b|x-y| \leq 3 b r$ and $|\gamma| \subset \overline{\mathrm{B}}(u ; 2 b r) \subset a \overline{\mathrm{B}}(x)$. Thus for all $z \in|\gamma|, d(z) \geq r / 2 b$, so

$$
\ell(\gamma[x, z]) \wedge \ell(\gamma[y, z]) \leq \frac{1}{2} \ell(\gamma) \leq \frac{3 b r}{2} \leq 3 b^{2} d(z)
$$




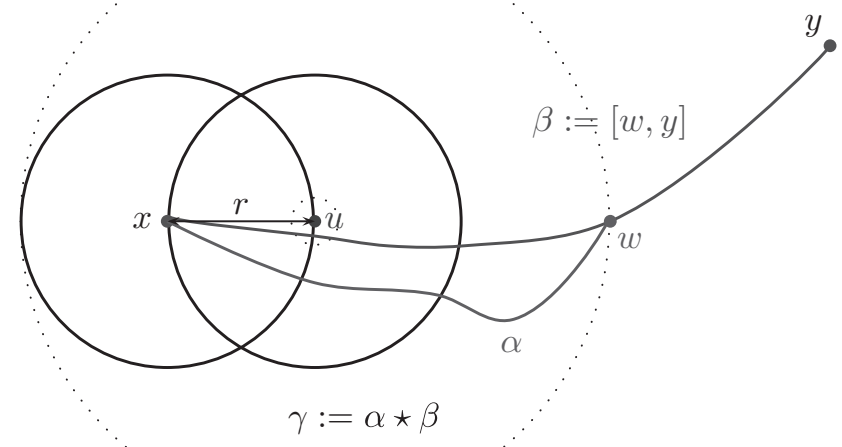

FIGURE 1. Using annular quasiconvexity

and we see that $\gamma$ is a double $3 b^{2}$-cone path.

Now suppose $y \notin \overline{\mathrm{B}}(u ; 2 r)$. See Figure 1 . Fix a geodesic $[x, y]$ from $x$ to $y$, let $w$ be the last point of $[x, y]$ in $\overline{\mathrm{B}}(u ; 2 r)$, and put $\beta:=[w, y] \subset[x, y]$. By the first case there is a path $\alpha$ joining $x, w$ in $\mathrm{A}(u ; r / b, 2 b r) \subset a \overline{\mathrm{B}}(x)$ with

$$
\ell(\alpha) \leq b|x-w| \leq 3 b r \quad \text { and } \quad \forall z \in|\alpha|, d(z) \geq r / 2 b .
$$

Let $\gamma:=\alpha \star \beta$. Then

$$
\ell(\gamma)=\ell(\alpha)+\ell(\beta) \leq 3 b r+|w-y| \leq 3 b|x-y| \leq 3 a b r .
$$

To check the double cone condition, we observe that:

$$
z \in|\alpha| \Rightarrow d(z) \geq r / 2 b, \quad \text { so } \quad \ell(\gamma[x, z]) \leq \ell(\alpha) \leq 3 b r \leq 6 b^{2} d(z) ;
$$

and since $|\beta| \cap \overline{\mathrm{B}}(u ; 2 r)=\{w\}$,

$$
z \in|\beta| \Rightarrow d(z)=\operatorname{dist}(z, A) \geq \operatorname{dist}(w, A) \geq 3 r / 2,
$$

so

$$
\ell(\gamma[x, z]) \wedge \ell(\gamma[y, z]) \leq \frac{1}{2} \ell(\gamma) \leq \frac{3 a b r}{2} \leq \operatorname{abd}(z)
$$

2.2.3. Plump Metric Spaces. A non-complete locally complete metric space $U$ is c-plump, $c \geq 1$, provided for each $x \in U$ and all $r \in(0, \operatorname{diam}(U))$

$$
\exists z \in \overline{\mathrm{B}}(x ; r) \quad \text { with } \quad d(z):=\operatorname{dist}(z, \partial U) \geq r / c .
$$


This terminology was introduced by Väisälä (see [27]) and perhaps is best understood when $U$ is an open subspace of a length space $X$, for then (2.6) asserts that $\operatorname{dist}(z, X \backslash U) \geq r / c$, so the open ball $\mathrm{B}(z ; r / c)$, in $X$, is contained in $U$. (Note that this may not hold if $X$ is not a length space.) The plumpness of $U$ is quantitatively equivalent to $\partial U$ being porous in $\bar{U}$ in the following sense: If $\partial U$ is $c$-porous in $\bar{U}$, then $U$ is $c$-plump. If $U$ is $c$-plump, then for each $b>c, \partial U$ is $b$-porous in $\bar{U}$.

The following results are straightforward to prove.

REMARK 2.7. Let $U$ be a non-complete locally complete metric space.

(a) If (2.6) holds for each $x \in U$ and each $r \in(0, \operatorname{diam}(U) / 2)$, then $U$ is $2 c$-plump.

(b) If $U$ is $c$-plump, then (2.6) also holds for $x \in \bar{U}$ and $r \in \Delta(U)$.

(c) If (2.6) holds for each $x \in \partial U$ and $r \in(0, \operatorname{diam}(\partial U))$, then $U$ is $6 c$ plump.

Here is an analog of [27, Lemma 2.14].

Lemma 2.8. Let $U$ be a c-plump metric space. Fix points $x, y \in U$. Put $R:=|x-y|>0$ and $R_{n}:=R / 2^{n}$. There exists points $x_{n} \in \overline{\mathrm{B}}\left(x ; R_{n}\right)$ and $y_{n} \in \overline{\mathrm{B}}\left(y ; R_{n}\right)$ such that

$$
d\left(x_{n}\right) \geq R_{n} / c, \quad d\left(y_{n}\right) \geq R_{n} / c,
$$

and $x_{n}, x_{n+1}$ and $y_{n}, y_{n+1}$ and $x_{0}, y_{0}$ are respectively $3 c$-proximate.

Proof. Since $R \in \Delta(U)$ and $U$ is $c$-plump, there are points $x_{n} \in \overline{\mathrm{B}}\left(x ; R_{n}\right)$ and $y_{n} \in \overline{\mathrm{B}}\left(y_{n} ; R_{n}\right)$ with $d\left(x_{n}\right) \geq R_{n} / c$ and $d\left(y_{n}\right) \geq R_{n} / c$. Then

$$
\left|x_{n}-x_{n+1}\right| \leq R_{n}+R_{n+1}=\frac{3}{2} R_{n}=3 R_{n+1} \leq 3 c\left[d\left(x_{n}\right) \wedge d\left(x_{n+1}\right)\right],
$$

so $x_{n}$ and $x_{n+1}$, and likewise $y_{n}$ and $y_{n+1}$, are $3 c$-proximate. Also, $\left|x_{0}-y_{0}\right| \leq\left|x-x_{0}\right|+|x-y|+\left|y-y_{0}\right| \leq 3 R=3 R_{0} \leq 3 c\left[d\left(x_{0}\right) \wedge d\left(y_{0}\right)\right]$, so $x_{0}$ and $y_{0}$ are $3 c$-proximate.

\section{Pointed Gromov-Hausdorff Tangent Spaces}

Here we recall the notion of pointed Gromov-Hausdorff distance, mention some basic properties, define the notion of tangent spaces and subspaces, and provide two examples. 


\subsection{Gromov-Hausdorff Distance}

A pointed metric space is a triple $(X, d ; a)$, that we often abbreviate as $(X ; a)$ when the distance is understood, where $(X, d)$ is a metric space and $a$ is a fixed base-point in $X$. Maps between pointed spaces are assumed to preserve base-points; thus $f:(X ; a) \rightarrow(Y ; b)$ means in particular that $f(a)=b$.

A distance function $\delta$ on the disjoint union $X \sqcup Y$ of two metric spaces is admissible if its restriction to each of $X, Y$ agrees with their original distances. Given $t>0$ and points $a \in X$ and $b \in Y$, we say that $\delta: X \sqcup Y \times X \sqcup Y \rightarrow$ $[0, \infty)$ is $(t ; a, b)$-admissible provided it is an admissible distance on $X \sqcup Y$ and

$$
\delta(a, b)<t, \quad \bar{B}_{\delta}\left(a ; t^{-1}\right) \subset N_{\delta}[Y ; t], \quad \bar{B}_{\delta}\left(b ; t^{-1}\right) \subset N_{\delta}[X ; t] .
$$

Following Gromov, we define the pointed Gromov-Hausdorff distance between two pointed metric spaces $(X ; a)$ and $(Y ; b)$ via

$$
\operatorname{dist}_{\mathscr{G} \mathscr{H}_{*}}((X ; a),(Y ; b)):=(1 / 2) \wedge \operatorname{dist}_{\mathscr{G} \mathscr{H}_{*}}((X ; a),(Y ; b))
$$

where

$$
\begin{aligned}
\operatorname{dist}_{\mathscr{G} \mathscr{H}_{*}}((X ; a),(Y ; b)) \\
\quad:=\inf \{t>0 \mid \exists \mathrm{a}(t ; a, b) \text {-admissible distance } \delta \text { on } X \sqcup Y\} .
\end{aligned}
$$

The quantity $\operatorname{dist}_{\operatorname{CH}_{*}}((X ; a),(Y ; b))$ is easily seen to be non-negative and symmetric. In addition, the triangle inequality holds provided at least two of the quantities in question are small enough.

The above definition of dist $\mathscr{G H}_{*}((X ; a),(Y ; b))$ is due to Gromov ${ }^{\dagger}$. In fact, dist $\mathscr{G H}_{*}$ is a distance function on the collection $\mathscr{G}_{\mathscr{H}_{*}}$ of all isometry classes of pointed proper metric spaces. We define pointed Gromov-Hausdorff convergence in the usual way: a sequence $\left(\left(X_{n} ; a_{n}\right)\right)_{n=1}^{\infty}$ of pointed metric spaces Gromov-Hausdorff converges to $\left(X_{\infty} ; a_{\infty}\right)$ provided

$$
\lim _{n \rightarrow \infty} \operatorname{dist} \mathscr{G H}_{*}\left(\left(X_{n} ; a_{n}\right),\left(X_{\infty} ; a_{\infty}\right)\right)=0 ;
$$

we denote this by writing

$$
\left(X_{n} ; a_{n}\right) \stackrel{\mathrm{GH}_{*}}{\longrightarrow}\left(X_{\infty} ; a_{\infty}\right) .
$$

Now we collect some information that we require in the sequel. The following is well known in the compact non-pointed setting (cf. [3], [26], [14]); see [17] for a detailed proof in the pointed category.

\footnotetext{
† Gromov [14, p. 63] calls this "modified Hausdorff distance" and credits it to O. Gabber.
} 
Embedding Theorem. Let $\left(\left(X_{n}, d_{n} ; a_{n}\right)\right)_{n=1}^{\infty}$ be a sequence of pointed proper metric spaces. Suppose that

$$
\sum_{n=1}^{\infty} \operatorname{dist} \mathscr{G}_{*}\left(\left(X_{n}, d_{n} ; a_{n}\right),\left(X_{n+1}, d_{n+1} ; a_{n+1}\right)\right)<\infty .
$$

Then there exists a non-complete locally complete metric space $\left(Y, d_{\infty}\right)$ and a point $a_{\infty}$ in $X_{\infty}:=\partial Y$ with the following properties:

(a) for each $n$ the space $\left(X_{n}, d_{n}\right)$ naturally isometrically embeds into $\left(Y, d_{\infty}\right)$,

(b) the space $\left(\bar{Y}, d_{\infty}\right)$ is proper (of course, $\bar{Y}=Y \cup \partial Y=Y \cup X_{\infty}$ ), and

(c) $\left(X_{\infty}, d_{\infty} ; a_{\infty}\right)$ is the pointed Gromov-Hausdorff limit of $\left(\left(X_{n}, d_{n} ; a_{n}\right)\right)_{n=1}^{\infty}$.

Moreover, if in addition each $\left(X_{n}, d_{n}\right)$ is a length space, then so is $\left(X_{\infty} ; d_{\infty}\right)$ and in this setting

(d) $\forall R>0$ : as $n \rightarrow \infty, \bar{B}_{d_{\infty}}\left(a_{n} ; R\right) \cap X_{n} \stackrel{\mathrm{H}}{\longrightarrow} \bar{B}_{d_{\infty}}\left(a_{\infty} ; R\right) \cap X_{\infty}$;

i.e., for each fixed radius, there is ordinary Hausdorff convergence, in $\bar{Y}$, of balls centered at the base-points.

In fact, the space $Y$ is simply the disjoint union $\bigsqcup_{1}^{\infty} X_{n}$ with an appropriate distance function defined on it.

In particular we note that a pointed Gromov-Hausdorff limit of complete uniformly doubling length spaces is a complete doubling length space (and hence geodesic).

For the record, here is a version of the compactness result for pointed proper spaces. See [14, p. 64].

Gromov's Compactness Theorem. The metric space $\left(\mathscr{G}_{\mathcal{H}_{*}}\right.$, dist $\left.\mathscr{G H}_{*}\right)$ is complete, and a collection $\mathscr{X}$ of (isometry classes of) pointed proper metric spaces is precompact in $\mathscr{G}_{*}$ if and only if there exists $(0, \infty) \stackrel{\nu}{\rightarrow}(0, \infty)$ such that

$$
\forall \varepsilon>0, \forall(X, d ; a) \in \mathscr{X}, \quad N\left(\varepsilon ; \bar{B}_{d}(a ; 1 / \varepsilon)\right) \leq v(\varepsilon) .
$$

We need some information regarding sequences in the space $Y:=\bigsqcup_{1}^{\infty} X_{n}$ constructed in the Embedding Theorem. In the sequel, the notation $\left(x_{n}\right)_{1}^{\infty} \subset$ $\bigsqcup_{1}^{\infty} X_{n}$ means that $\left(x_{n}\right)_{1}^{\infty}$ is a sequence in $Y$ and that for each $n \in \mathrm{N}, x_{n} \in X_{n}$.

FACT 3.1. Let $\left(y_{n}\right)_{n=1}^{\infty}$ be a sequence in $Y:=\bigsqcup_{1}^{\infty} X_{n}$ (the space constructed in the Embedding Theorem) that converges to some point $y \in \bar{Y}$. Suppose there are strictly increasing sequences $\left(n_{k}\right)_{k=1}^{\infty},\left(m_{k}\right)_{k=1}^{\infty}$ in $\mathrm{N}$ such that for all $k \in \mathrm{N}, y_{n_{k}} \in X_{m_{k}}$. Then $y \in X_{\infty}$. 
We also need to know that Lipschitz maps induce Lipschitz maps on pointed Gromov-Hausdorff limits as indicated below. Basically, this is a consequence of the Arzela-Ascoli theorem; see [11, Lemma 8.20] or [17].

FACT 3.2. Suppose $\left(X_{n} ; a_{n}\right) \stackrel{f_{n}}{\longrightarrow}\left(Y_{n} ; b_{n}\right)$ are uniformly Lipschitz maps between pointed proper metric spaces that pointed Gromov-Hausdorff converge to $\left(X_{\infty}, a_{\infty}\right)$ and $\left(Y_{\infty} ; b_{\infty}\right)$ respectively. Then there exist a Lipschitz map $f_{\infty}:\left(X_{\infty} ; a_{\infty}\right) \rightarrow\left(Y_{\infty} ; b_{\infty}\right)$ and a subsequence $\left(f_{n_{k}}\right)_{k=1}^{\infty}$ of $\left(f_{n}\right)_{n=1}^{\infty}$ such that $\left(f_{n_{k}}\right)_{k=1}^{\infty}$ converges locally uniformly to $f_{\infty}$.

\subsection{Tangent Spaces, Tangent Subspaces, and Examples}

Let $X$ be a complete doubling metric space. Then for every $\sigma>0, \sigma X:=$ $(X, \sigma|\cdot|)$ is also doubling with the same doubling parameter. Let $\left(a_{n}\right)_{1}^{\infty}$ be any sequence in $X$ and $\left(\tau_{n}\right)_{1}^{\infty}$ a sequence in $\Delta(X)$. Put $X_{n}:=\tau_{n}^{-1} X:=\left(X, d_{n}\right)$, where $d_{n}:=\tau_{n}^{-1}|\cdot|$. A simple application of Gromov's Compactness Theorem reveals that $\left(\left(X_{n} ; a_{n}\right)\right)_{1}^{\infty}$ subconverges with respect to pointed GromovHausdorff distance. We write $\operatorname{Tan}(X)$ to denote the collection of all such limits, each of which is a pointed complete doubling space and called a pointed tangent space of $X$.

Next we describe the collections $\operatorname{Tan}(X ; A)$ and $\operatorname{Tan}_{b}(X, A)$ that, roughly speaking, consist of certain "pointed tangent subspaces of tangent spaces" $\left(X_{\infty}, A_{\infty} ; a_{\infty}\right)$ with $a_{\infty} \in A_{\infty} \subset X_{\infty}$. Let $A$ be a non-empty closed subspace of a complete doubling metric space $X$; so $A$ itself is complete and doubling. Let $\left(a_{n}\right)_{1}^{\infty}$ be a sequence in $A$ and $\left(\tau_{n}\right)_{1}^{\infty}$ a sequence in $\Delta(X)$. As above, $X_{n}:=\tau_{n}^{-1} X:=\left(X, d_{n}\right)$, with $d_{n}:=\tau_{n}^{-1}|\cdot|$, and $A_{n}:=\tau_{n}^{-1} A \subset X_{n}$. Passing to an appropriate subsequence, we may assume that

$$
\begin{aligned}
& \sum_{n=1}^{\infty} \operatorname{dist}_{\mathscr{G} \mathscr{H}_{*}}\left(\left(X_{n} ; a_{n}\right),\left(X_{n+1} ; a_{n+1}\right)\right)<\infty, \\
& \sum_{n=1}^{\infty} \operatorname{dist}_{\mathscr{H} \mathscr{H}_{*}}\left(\left(A_{n} ; a_{n}\right),\left(A_{n+1} ; a_{n+1}\right)\right)<\infty .
\end{aligned}
$$

Appealing to the Embedding Theorem we can assert that as $n \rightarrow \infty$ :

$$
\begin{aligned}
& \left(X_{n} ; a_{n}\right) \stackrel{\mathrm{GH}_{*}}{\longrightarrow}\left(X_{\infty} ; x_{\infty}\right) \quad \text { where } X_{\infty}:=\partial Y \text { and } Y:=\bigsqcup_{n=1}^{\infty} X_{n}, \\
& \left(A_{n} ; a_{n}\right) \stackrel{\mathrm{GH}_{*}}{\longrightarrow}\left(A_{\infty} ; a_{\infty}\right) \quad \text { where } A_{\infty}:=\partial Z \text { and } Z:=\bigsqcup_{n=1}^{\infty} A_{n} .
\end{aligned}
$$


Here $Y$ and $Z$ come with distances $d_{Y}$ and $d_{Z}$ which are defined by 'chaining' certain admissible distances on $X_{n} \sqcup X_{n+1}$ and $A_{n} \sqcup A_{n+1}$ respectively. See [17].

We claim that there is a closed subspace $\tilde{A}_{\infty} \subset X_{\infty}$ such that $\left(A_{\infty} ; a_{\infty}\right)$ is isometric to $\left(\tilde{A}_{\infty} ; x_{\infty}\right)$. With this fact in hand, we define $\operatorname{Tan}(X ; A)$ to be the collection of all such triples $\left(X_{\infty}, A_{\infty} ; a_{\infty}\right)$ where we view $a_{\infty} \in A_{\infty} \subset X_{\infty}$. And then $\operatorname{Tan}_{\mathrm{b}}(X, A)$ is the subcollection of $\operatorname{Tan}(X ; A)$ determined by the additional requirement that the original sequence $\left(a_{n}\right)_{1}^{\infty}$ lies in $\operatorname{bd}(A)$.

We note that when $A \subset \mathrm{R}^{n}, \operatorname{Tan}\left(\mathrm{R}^{n}, A\right)$ can be identified with $\operatorname{Tan}(A)$.

For the readers convenience, we confirm the above claim. First, let $b_{\infty} \in$ $A_{\infty}=\partial Z$ and suppose $\left(b_{n}\right)_{1}^{\infty},\left(c_{n}\right)_{1}^{\infty}$ are any two sequences in $Z$ with $b_{n}, c_{n} \in$ $A_{n}$ for all $n, d_{Z}\left(b_{n}, b_{\infty}\right) \rightarrow 0, d_{Z}\left(c_{n}, b_{\infty}\right) \rightarrow 0$, and with $\left(b_{n}\right)_{1}^{\infty},\left(c_{n}\right)_{1}^{\infty}$ also convergent in $\bar{Y}$. Then

$$
d_{Y}\left(b_{n}, c_{n}\right)=d_{n}\left(b_{n}, c_{n}\right)=d_{Z}\left(b_{n}, c_{n}\right) \leq d_{Z}\left(b_{n}, b\right)+d_{Z}\left(c_{n}, b\right) \rightarrow 0,
$$

so in fact $\left(b_{n}\right)_{1}^{\infty},\left(c_{n}\right)_{1}^{\infty}$ also have the same limit in $\bar{Y}$.

We define a distance preserving map $\left(A_{\infty} ; a_{\infty}\right) \stackrel{f}{\hookrightarrow}\left(X_{\infty} ; x_{\infty}\right)$ as follows. We begin by setting $f\left(a_{\infty}\right):=x_{\infty}$. Next, let $b_{\infty} \in A_{\infty}=\partial Z$. Choose any sequence $\left(b_{n}\right)_{1}^{\infty}$ in $Z$ with $b_{n} \in A_{n}$ for all $n$ and $d_{Z}\left(b_{n}, b_{\infty}\right) \rightarrow 0$. We show below that $\left(b_{n}\right)_{1}^{\infty}$ converges to some $y_{\infty} \in X_{\infty}$. The previous paragraph explains why this limit is independent of the choice of the sequence $\left(b_{n}\right)_{1}^{\infty}$. Thus we may define $f\left(b_{\infty}\right):=y_{\infty}$.

Using the information that $\left(b_{n}\right)_{1}^{\infty}$ converges to $b_{\infty}$ in $\bar{Z}$ and that $\left(X_{n} ; a_{n}\right)$ Gromov-Hausdorff converges to $\left(X_{\infty} ; x_{\infty}\right)$, it is straightforward to find an $R>0$ such that for all sufficiently large $n, b_{n} \in \overline{\mathrm{B}}_{\infty}\left(x_{\infty} ; R\right) \subset \bar{Y}$. (For instance, one can take $R:=d_{Z}\left(b_{\infty}, a_{\infty}\right)+2$.) Since $\bar{Y}$ is proper, it follows that $\left(b_{n}\right)_{1}^{\infty}$ subconverges to some point, say $y_{\infty}$, of $Y$. According to Fact 3.1, $y_{\infty} \in X_{\infty}$. In fact, using the work two paragraphs above we easily see that every convergent subsequence of $\left(b_{n}\right)_{1}^{\infty}$ must also have limit $y_{\infty}$, thus $\left(b_{n}\right)_{1}^{\infty}$ itself converges to $y_{\infty}$.

Finally, to see that $f$ preserves distances, let $b_{\infty}, c_{\infty} \in A_{\infty}$. Put $y_{\infty}:=$ $f\left(b_{\infty}\right), z_{\infty}:=f\left(c_{\infty}\right)$. By definition, there are sequences $\left(b_{n}\right)_{1}^{\infty},\left(c_{n}\right)_{1}^{\infty}$ in $Z$ with $b_{n}, c_{n} \in A_{n}$ for all $n$ and such that

$$
\begin{array}{ll}
b_{n} \rightarrow b_{\infty} \text { in } \bar{Z}, & b_{n} \rightarrow y_{\infty} \text { in } \bar{Y}, \\
c_{n} \rightarrow c_{\infty} \text { in } \bar{Z}, & c_{n} \rightarrow z_{\infty} \text { in } \bar{Y} .
\end{array}
$$

Thus

$$
\begin{aligned}
d_{Y}\left(y_{\infty}, z_{\infty}\right) & =\lim _{n \rightarrow \infty} d_{Y}\left(b_{n}, c_{n}\right)=\lim _{n \rightarrow \infty} d_{n}\left(b_{n}, c_{n}\right) \\
& =\lim _{n \rightarrow \infty} d_{Z}\left(b_{n}, c_{n}\right)=d_{Z}\left(b_{\infty}, c_{\infty}\right) .
\end{aligned}
$$


To recap, to say that $\left(X_{\infty}, A_{\infty} ; a_{\infty}\right) \in \operatorname{Tan}_{\mathrm{b}}(X, A)$ means that there are sequences $\left(a_{n}\right)_{1}^{\infty}$ in $\operatorname{bd}(A)$ and $\left(\tau_{n}\right)_{1}^{\infty}$ in $\Delta(X)$ such that $\left(\tau_{n}^{-1} X ; a_{n}\right)$ and $\left(\tau_{n}^{-1} A ; a_{n}\right)$ have pointed Gromov-Hausdorff limits $\left(X_{\infty} ; a_{\infty}\right)$ and $\left(A_{\infty} ; a_{\infty}\right)$, respectively, with $a_{\infty} \in A_{\infty} \subset X_{\infty}$; since $A_{\infty}$ is complete, it is a closed subspace of $X_{\infty}$.

We close this subsection with two illustrative examples. It is not difficult, for example by using the Arzela-Ascoli theorem (cf. [8, 2.3(iv), p. 35; 2.5.14, p. 47] or $[6,1.23$, p. $14 ; 3.10$, p. 36]), to show that every pointed tangent space of a $c$-quasiconvex proper metric space is also $c$-quasiconvex. However, some care is required as indicated by the following. (An alternative argument could use the facts that: every quasiconvex space is bilipschitz equivalent to a length space, pointed tangents of length spaces are also length spaces, and bilipschitz maps induce bilipschitz maps at the tangent level.)

ExAmple 3.3. There is a quasiconvex open subspace $U$ of $\mathrm{R}^{2}$ such that for some $\left(X_{\infty}, A_{\infty} ; a_{\infty}\right)$ in $\operatorname{Tan}\left(\mathrm{R}^{2}, \mathrm{R}^{2} \backslash U\right), U_{\infty}:=X_{\infty} \backslash A_{\infty}$ is not quasiconvex.

PROOF. For each $m, n \in \mathrm{N}$, let $a_{n}:=0, \tau_{n}:=2^{-2 n}$,

$$
C_{m}:=\left\{\frac{k}{m 2^{m}} \mid k \in\left[0, m 2^{m}\right] \cap \mathrm{Z}\right\}, \quad B_{m}:=2^{-m} C_{m}+2^{-m},
$$

and put $A_{n}:=\tau_{n}^{-1} A$ where

$$
A:=\{(0,0)\} \cup \bigcup_{m=1}^{\infty}\left(B_{m} \times\{0\}\right) \subset[0,1] \times\{0\} \subset \mathrm{R}^{2} .
$$

It is easy to check that $U:=\mathrm{R}^{2} \backslash A$ is quasiconvex. Also, for each $R>0$, $A_{n} \cap \overline{\mathrm{B}}\left(a_{n} ; R\right)$ Hausdorff converges to $[0, R] \times\{0\}$ in $\mathrm{R}^{2}$, so $\left(A_{n} ; a_{n}\right)$ pointed Gromov-Hausdorff converges to $\left(A_{\infty} ; a_{\infty}\right):=([0, \infty) \times\{0\}, 0)$. Clearly $U_{\infty}:=\mathrm{R}^{2} \backslash A_{\infty}$ fails to be quasiconvex.

CONSTRUCTION FOR Example E. We demonstrate that any non-empty closed subset of $\mathrm{R}^{n}$ is a tangent subspace for some 1-bounded turning 1-linearly locally connected open subset of $\mathrm{R}^{n}$. This is based on two simple facts: First, any set in $\mathrm{R}^{n}$ can be approximated, with respect to dist $\mathscr{G}_{\mathscr{H}_{*}}$, by a sequence of compact totally disconnected sets. Second, the complement of a closed totally disconnected set in $\mathrm{R}^{n}$ is 1-bounded turning and 1-linearly locally connected; see [15, Proposition 4.1, Corollary 4.2].

Let $C \subset \mathrm{R}^{n}$ be closed and assume $0 \in C$. For each $k \in \mathrm{N}$, let

$$
B_{k}:=C_{k} \cap k \overline{\mathrm{B}}^{n} \quad \text { where } \quad C_{k}:=\left\{z \in \mathrm{Z}^{n} \mid \operatorname{dist}(z, C)<1 / k\right\}
$$


and put $U:=\mathrm{R}^{n} \backslash A$ where $A:=\bigcup_{k=1}^{\infty}\left(a_{k}+B_{k}\right)$ and $a_{k}:=\left(2^{k}, 0, \ldots, 0\right) \in$ $\mathrm{R}^{n}$. Since $A$ is closed and totally disconnected, $U$ is 1 -bounded turning and 1-linearly locally connected.

It is easy to see that $C_{k} \stackrel{\mathrm{H}}{\longrightarrow} C$ and that $\left(B_{k} ; 0\right) \stackrel{\mathrm{GH}_{*}}{\longrightarrow}(C ; 0)$. Taking $A_{k}:=A=\mathrm{R}^{n} \backslash U$ (and using the scalings $\tau_{k}=1:-$ ), it is not difficult to check that $\left(A_{k} ; a_{k}\right)$ Gromov-Hausdorff converges to $(C ; 0)$, so $(C ; 0) \in \operatorname{Tan}\left(\mathrm{R}^{n} \backslash U\right)$.

\section{Proofs of Main Results}

Here we establish the results announced in the introduction. As mentioned there, the various necessary conditions can be strengthened. We explicitly indicate this for Proposition B (see Proposition 4.2) but only describe it in the actual proofs for Theorems A and D.

We begin with Theorem D, proceed with Propositions B and C, and then turn to Theorem A. Our arguments for the latter mimic Väisälä's; see the proofs of [27, Theorems $2.15,3.4,3.6]$. For the reader's convenience, we supply all the details.

Everywhere in this section $X$ is assumed to be (at least) a complete doubling metric space. We remind the reader that such a space is proper, hence (by the Hopf-Rinow theorem) also geodesic whenever it is a length space. These properties are inherited by tangent spaces. Similar comments apply if we start with a quasiconvex complete doubling space: it is bilipschitz equivalent to a length (hence geodesic) space, so by Fact 3.2 its tangents also possess this property and hence are quasiconvex too. See also the paragraph just before Example 3.3.

Frequently, in our arguments, we are given a pointed tangent space $\left(X_{\infty}\right.$, $\left.a_{\infty}\right)$ in $\operatorname{Tan}(X)$ or $\left(X_{\infty}, A_{\infty} ; a_{\infty}\right)$ in $\operatorname{Tan}_{\mathrm{b}}(X, A)$. Recall from $\$ 3.2$ that this means that there are sequences $\left(a_{n}\right)_{1}^{\infty}$ in $X$, or in $\operatorname{bd}(A)$, and $\left(\tau_{n}\right)_{1}^{\infty}$ in $\Delta(X)$ such that with $X_{n}:=\tau_{n}^{-1} X:=\left(X, d_{n}\right), d_{n}:=\tau_{n}^{-1}|\cdot|$, and $A_{n}:=\tau_{n}^{-1} A \subset X_{n}$ we have

$$
\begin{gathered}
\left(X_{n} ; a_{n}\right) \stackrel{\mathrm{GH}_{*}}{\longrightarrow}\left(X_{\infty} ; a_{\infty}\right) \text { and }\left(A_{n} ; a_{n}\right) \stackrel{\mathrm{GH}_{*}}{\longrightarrow}\left(A_{\infty} ; a_{\infty}\right) \text { as } n \rightarrow \infty, \\
\text { where } a_{\infty} \in A_{\infty} \subset X_{\infty}:=\partial Y \text { and } Y:=\bigsqcup_{n=1}^{\infty} X_{n} \supset \bigsqcup_{n=1}^{\infty} A_{n} .
\end{gathered}
$$

Here $\bar{Y}$ is equipped with a distance $d_{\infty}$ that satisfies $\left.d_{\infty}\right|_{X_{n} \times X_{n}}=d_{n}$ (i.e., for all $\left.x, y \in X_{n}, d_{\infty}(x, y)=d_{n}(x, y)=\tau_{n}^{-1}|x-y|\right)$, and $\left(\bar{Y}, d_{\infty}\right)$ is a proper metric space.

Conversely, if we start with sequences $\left(a_{n}\right)_{1}^{\infty}$ in $X$, or in $\operatorname{bd}(A)$, and $\left(\tau_{n}\right)_{1}^{\infty}$ in $\Delta(X)$, then by Gromov's Compactness Theorem in conjunction with the 
Embedding Theorem we may pass to a subsequence and obtain similar statements.

We tacitly make use of these ideas and notations, but do not repeat the above discussion.

\subsection{Proof of Theorem D}

We first demonstrate that annular quasiconvexity is inherited by tangents. We assume $X$ is a $c$-annular quasiconvex complete doubling space. Suppose we are given a pointed tangent space $\left(X_{\infty} ; a_{\infty}\right) \in \operatorname{Tan}(X)$. We show that $X_{\infty}$ is $b$ annular quasiconvex at $a_{\infty}$ where $b=45 c$; in particular, $a_{\infty}$ is not a cut-point of $X_{\infty}$.

According to Fact 2.2(a), $X$ is quasiconvex. Hence so is $X_{\infty}$; see the discussion immediately above. In particular, $X_{\infty}$ is connected.

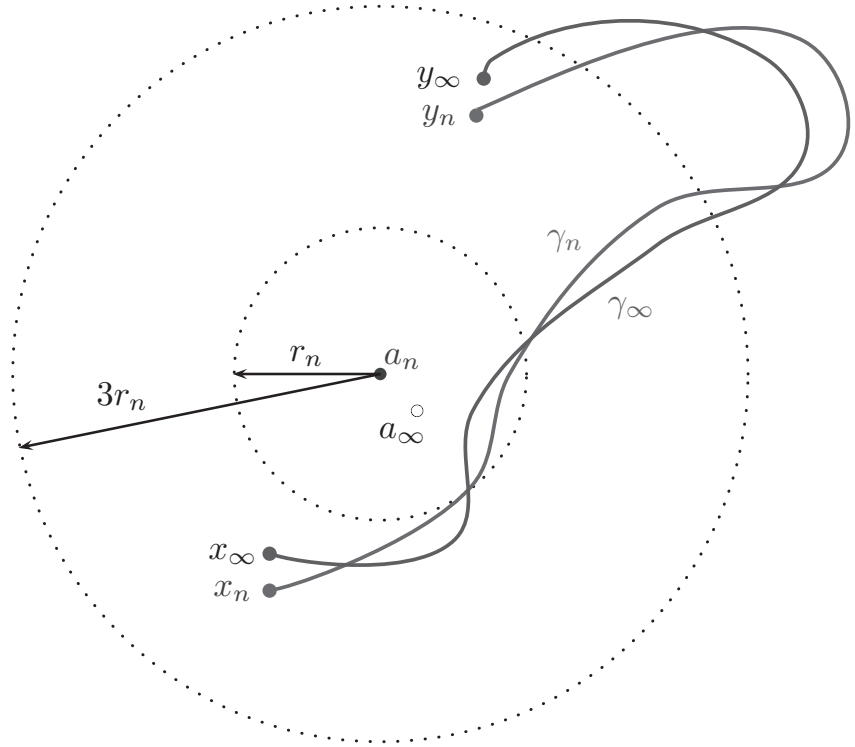

FIGURE 2. Annular quasiconvexity in tangents

Fix $r>0$ and points $x_{\infty}, y_{\infty}$ in $\mathrm{A}_{\infty}\left(a_{\infty} ; r, 2 r\right) \subset X_{\infty}$. See Figure 2. Choose sequences $\left(a_{n}\right)_{1}^{\infty},\left(x_{n}\right)_{1}^{\infty},\left(y_{n}\right)_{1}^{\infty}$ in $\bigsqcup_{1}^{\infty} X_{n}$ that converge in $\bar{Y}$ to $a_{\infty}, x_{\infty}, y_{\infty}$ respectively. Assuming, e.g., that $d_{\infty}\left(x_{n}, x_{\infty}\right) \wedge d_{\infty}\left(y_{n}, y_{\infty}\right) \wedge d_{\infty}\left(a_{n}, a_{\infty}\right)<$ $r / 8$ we find that $x_{n}, y_{n} \in \mathrm{A}\left(a_{n} ; r_{n}, 3 r_{n}\right)$ where $r_{n}:=\tau_{n}(3 r / 4)$.

Appealing to Fact 2.2(a,c) we obtain $b$-quasiconvex paths $\gamma_{n}$ that join the points $x_{n}, y_{n}$ in $\mathrm{A}\left(a_{n} ; r_{n} / 9 c, 27 c r_{n}\right)$. Let $\gamma_{n}$ be parameterized by arclength. Then they are 1-Lipschitz, so $\left(\gamma_{n}\right)_{1}^{\infty}$ is an equicontinuous sequence of paths in a compact subspace of $\bar{Y}$. Hence the Arzela-Ascoli theorem (cf. [8, 2.3(iv), 
p. 35; 2.5.14, p. 47] or $[6,1.23$, p. $14 ; 3.10$, p. 36]) provides a subsequence of $\left(\gamma_{n}\right)_{1}^{\infty}$ that converges uniformly to a rectifiable path $\gamma_{\infty}$ that joins $x_{\infty}, y_{\infty}$ in $X_{\infty}$.

Now

$$
\ell_{\infty}\left(\gamma_{\infty}\right)=\lim _{n \rightarrow \infty} \ell_{\infty}\left(\gamma_{n}\right) \leq b \lim _{n \rightarrow \infty} d_{\infty}\left(x_{n}, y_{n}\right)=b d_{\infty}\left(x_{\infty}, y_{\infty}\right)
$$

so $\gamma_{\infty}$ is a $b$-quasiconvex path joining $x_{\infty}, y_{\infty}$. Moreover,

$$
\frac{r_{n}}{9 c} \leq\left|\gamma_{n}(t)-a_{n}\right| \leq 27 c r_{n}, \quad \text { so } \quad \frac{r}{12 c} \leq d_{\infty}\left(\gamma_{n}(t), a_{n}\right) \leq 21 c r
$$

and thus $\left|\gamma_{\infty}\right| \subset A_{\infty}\left(a_{\infty} ; r / 12 c, 21 c r\right)$.

Conversely, suppose $X$ is a non-annular quasiconvex complete doubling length space. We exhibit a pointed tangent space $\left(X_{\infty}, a_{\infty}\right) \in \operatorname{Tan}(X)$ with $X_{\infty} \backslash\left\{a_{\infty}\right\}$ non-connected. The assumption that $X$ is not annular quasiconvex means that for each $n \in \mathrm{N}$ we can select base-points $a_{n} \in X$, radii $r_{n}>0$ and points $x_{n}, y_{n}$ in $\mathrm{A}\left(a_{n} ; r_{n}, 2 r_{n}\right)$ such that

(4.1) $x_{n}, y_{n}$ cannot be joined by an $n$-quasiconvex path in $\mathrm{A}\left(a_{n} ; r_{n} / n, 2 n r_{n}\right)$.

Using the scales $\tau_{n}:=r_{n}$ (so $X_{n}:=r_{n}^{-1} X$ ) we pass to a subsequence and obtain a pointed tangent space $\left(X_{\infty} ; a_{\infty}\right) \in \operatorname{Tan}(X)$. We claim that $X_{\infty} \backslash\left\{a_{\infty}\right\}$ is not connected; to prove this we assume otherwise and show that for large $n$ the condition (4.1) is violated.

So, assume $X_{\infty} \backslash\left\{a_{\infty}\right\}$ is connected; then it is piecewise-geodesically connected. Since $d_{\infty}\left(x_{n}, a_{n}\right), d_{\infty}\left(y_{n}, a_{n}\right) \in[1,2]$, we may (pass to another subsequence and) assume that $\left(x_{n}\right),\left(y_{n}\right)$ converge in $\bar{Y}$, respectively, to points $x_{\infty}, y_{\infty}$ that lie in $\mathrm{A}_{\infty}\left(a_{\infty} ; 1,2\right) \subset X_{\infty} \backslash\left\{a_{\infty}\right\}$. Select points $z_{0}:=x_{\infty}, z_{1}, \ldots$, $z_{m-1}, z_{m}:=y_{\infty}$ in $X_{\infty} \backslash\left\{a_{\infty}\right\}$ such that the piecewise geodesic path

$$
\gamma_{\infty}:=\left[z_{0}, z_{1}\right] \star\left[z_{1}, z_{2}\right] \star \cdots \star\left[z_{m-1}, z_{m}\right]
$$

joins $x_{\infty}, y_{\infty}$ in $X_{\infty} \backslash\left\{a_{\infty}\right\}$. See Figure 3 .

Set $\delta:=\operatorname{dist}_{\infty}\left(a_{\infty},\left|\gamma_{\infty}\right|\right), d:=d_{\infty}\left(x_{\infty}, y_{\infty}\right), \lambda:=\ell_{\infty}\left(\gamma_{\infty}\right)=$ $\sum_{i=1}^{m} d_{\infty}\left(z_{i}, z_{i-1}\right)$ and let $C:=(\lambda / d) \vee(1 / \delta)$. Then (see (2.1))

$$
\lambda \leq C d \text { and }\left|\gamma_{\infty}\right| \subset \overline{\mathrm{B}}_{\infty}\left(a_{\infty} ; 2(C+1)\right) \text { and }\left|\gamma_{\infty}\right| \cap \mathrm{B}_{\infty}\left(a_{\infty} ; 1 / C\right)=\emptyset,
$$

so $\gamma_{\infty}$ is a $C$-quasiconvex path joining $x_{\infty}, y_{\infty}$ in $A_{\infty}\left(a_{\infty} ; 1 / C, 2(C+1)\right)$.

Next, put $t:=(1 / 10)[d \wedge(1 / d) \wedge \delta \wedge(1 / \delta)]$. As $\left[z_{i-1}, z_{i}\right]$ is a geodesic, we may insert additional points without changing the value of $\delta$ or $d$ or $\lambda$. Thus we may assume that both

$$
m \geq 10 \vee C \quad \text { and } \quad \forall 1 \leq i \leq m, \quad d_{\infty}\left(z_{i}, z_{i-1}\right)<t / 10 .
$$




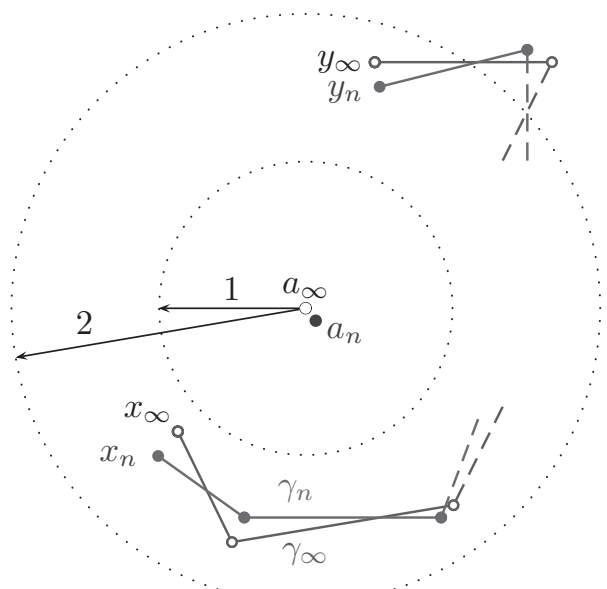

FIGURE 3. Piecewise geodesics $\gamma_{\infty}$ and $\gamma_{n}$

Since $\left(X_{n} ; a_{n}\right) \stackrel{\mathrm{GH}_{*}}{\longrightarrow}\left(X_{\infty} ; a_{\infty}\right)$ and $\left(x_{n}\right),\left(y_{n}\right),\left(a_{n}\right)$ converge in $\bar{Y}$ to $x_{\infty}, y_{\infty}$, $a_{\infty}$ respectively, we can select $n \in \mathrm{N}$ sufficiently large so that

$$
\begin{gathered}
n>2 C, \operatorname{dist}_{\mathscr{S O H}_{*}}\left(\left(X_{n} ; a_{n}\right),\left(X_{\infty}, a_{\infty}\right)\right)<t / 10 m \text { and } \\
d_{\infty}\left(x_{n}, x_{\infty}\right) \vee d_{\infty}\left(y_{n}, y_{\infty}\right) \vee d_{\infty}\left(a_{n}, a_{\infty}\right)<t / 10 m .
\end{gathered}
$$

Since $\lambda+\delta \leq C d+\delta \leq(C+1) / 10 t<2 m / 10 t$, for each $0 \leq i \leq m$,

$$
d_{\infty}\left(z_{i}, a_{\infty}\right) \leq \operatorname{dist}_{\infty}\left(a_{\infty},\left|\gamma_{\infty}\right|\right)+\ell_{\infty}\left(\gamma_{\infty}\right)=\delta+\lambda<m / 5 t .
$$

In particular, $z_{i} \in \mathrm{B}_{\infty}\left(a_{\infty} ; 10 m / t\right)$, so for each $1 \leq i<m$ there exist points $z_{n i} \in X_{n}$ with $d_{\infty}\left(z_{n i}, z_{i}\right)<t / 10 m$. Put $z_{n 0}:=x_{n}, z_{n m}:=y_{n}$ and set

$$
\gamma_{n}:=\left[z_{n 0}, z_{n 1}\right] \star\left[z_{n 1}, z_{n 2}\right] \star \cdots \star\left[z_{n, m-1}, z_{n m}\right] .
$$

See Figure 3. We claim that $\gamma_{n}$ is a $2 C$-quasiconvex path that joins $x_{n}, y_{n}$ in $\mathrm{A}\left(a_{n} ; r_{n} / 2 C, 2(2 C+1) r_{n}\right)$. Since $n \geq 2 C+1$, this directly contradicts (4.1).

To corroborate this claim, we first note that

$$
\begin{aligned}
d_{\infty}\left(z_{n i}, z_{n, i-1}\right) & \leq d_{\infty}\left(z_{n i}, z_{i}\right)+d_{\infty}\left(z_{i}, z_{i-1}\right)+d_{\infty}\left(z_{i-1}, z_{n, i-1}\right) \\
& \leq \frac{t}{10 m}+\frac{t}{10}+\frac{t}{10 m} .
\end{aligned}
$$

Thus for each $1 \leq i \leq m$ and all $z \in\left[z_{n i}, z_{n, i-1}\right]$ :

$$
d_{\infty}\left(z, z_{i}\right) \leq d_{\infty}\left(z, z_{n i}\right)+d_{\infty}\left(z_{n i}, z_{i}\right) \leq d_{\infty}\left(z_{n i}, z_{n, i-1}\right)+\frac{t}{10 m}<\frac{t}{10}+\frac{3 t}{10 m},
$$


so

$$
\begin{aligned}
\delta=\operatorname{dist}_{\infty}\left(a_{\infty},\left|\gamma_{\infty}\right|\right) & \leq d_{\infty}\left(a_{\infty}, z_{i}\right) \leq d_{\infty}\left(z_{i}, z\right)+d_{\infty}\left(z, a_{n}\right)+d_{\infty}\left(a_{n}, a_{\infty}\right) \\
& \leq \frac{t}{10}+\frac{3 t}{10 m}+d_{\infty}\left(z, a_{n}\right)+\frac{t}{10 m} \leq \frac{t}{5}+d_{\infty}\left(z, a_{n}\right)
\end{aligned}
$$

and therefore $d_{\infty}\left(z, a_{n}\right) \geq \delta-t / 5 \geq 9 \delta / 10$. We conclude that

$$
\operatorname{dist}_{\infty}\left(a_{n},\left|\gamma_{n}\right|\right) \geq 9 \delta / 10 \geq \delta / 2
$$

Next, we estimate $\ell_{\infty}\left(\gamma_{n}\right)$. Observe that

$$
\begin{aligned}
\left|d-d_{\infty}\left(x_{n}, y_{n}\right)\right| & =\left|d_{\infty}\left(x_{\infty}, y_{\infty}\right)-d_{\infty}\left(x_{n}, y_{n}\right)\right| \\
& \leq d_{\infty}\left(x_{n}, x_{\infty}\right)+d_{\infty}\left(y_{n}, y_{\infty}\right) \leq t / 5 m
\end{aligned}
$$

Thus $d_{\infty}\left(x_{n}, y_{n}\right) \geq d-t / 5 m \geq 10 t-t / 5 m \geq 9 t$, so

$$
\frac{t}{5}\left(1+\frac{C}{m}\right) \leq \frac{2 t}{5}<d_{\infty}\left(x_{n}, y_{n}\right)
$$

and then

$$
\begin{aligned}
\frac{t}{5}+C d & \leq \frac{t}{5}+C\left(d_{\infty}\left(x_{n}, y_{n}\right)+\frac{t}{5 m}\right)=\frac{t}{5}\left(1+\frac{C}{m}\right)+C d_{\infty}\left(x_{n}, y_{n}\right) \\
& \leq(C+1) d_{\infty}\left(x_{n}, y_{n}\right)
\end{aligned}
$$

and therefore

$$
\begin{aligned}
\ell_{\infty}\left(\gamma_{n}\right) & =\sum_{i=1}^{m} d_{\infty}\left(z_{n i}, z_{n, i-1}\right) \leq \sum_{i=1}^{m}\left(\frac{t}{5 m}+d_{\infty}\left(z_{i}, z_{i-1}\right)\right) \\
& =\frac{t}{5}+\ell_{\infty}\left(\gamma_{\infty}\right)=\frac{t}{5}+\lambda \leq \frac{t}{5}+C d \leq(C+1) d_{\infty}\left(x_{n}, y_{n}\right)
\end{aligned}
$$

Finally, since $\operatorname{dist}_{\infty}\left(a_{n},\left|\gamma_{n}\right|\right) \geq \delta / 2$, we deduce that

$$
\operatorname{dist}\left(a_{n},\left|\gamma_{n}\right|\right) \geq \frac{\delta}{2} r_{n} \geq \frac{r_{n}}{2 C}
$$

We conclude that $\gamma_{n}$ is a $2 C$-quasiconvex path in $\mathrm{A}\left(a_{n} ; r_{n} / 2 C, 2(2 C+1) r_{n}\right)$ that joins $x_{n}, y_{n}$. 


\subsection{Proof of Proposition $C$}

Assume $U$ is a non-complete locally complete $c$-plump metric space and $3 c$ proximate points in $U$ can be joined by $b$-uniform paths. We demonstrate that $U$ is $a$-uniform with $a:=6 b c(3 \vee 2 b)$.

Fix $x, y \in U$. Put $R:=|x-y|, R_{n}:=R / 2^{n}$, and select $x_{n} \in \overline{\mathrm{B}}\left(x ; R_{n}\right)$, $y_{n} \in \overline{\mathrm{B}}\left(y_{n} ; R_{n}\right)$ as in Lemma 2.8. The hypotheses provide $b$-uniform paths $\alpha_{n}$, $\beta_{n}, \gamma_{0}$ that join $x_{n}$ to $x_{n+1}, y_{n}$ to $y_{n+1}, x_{0}$ to $y_{0}$ respectively. See Figure 4 .

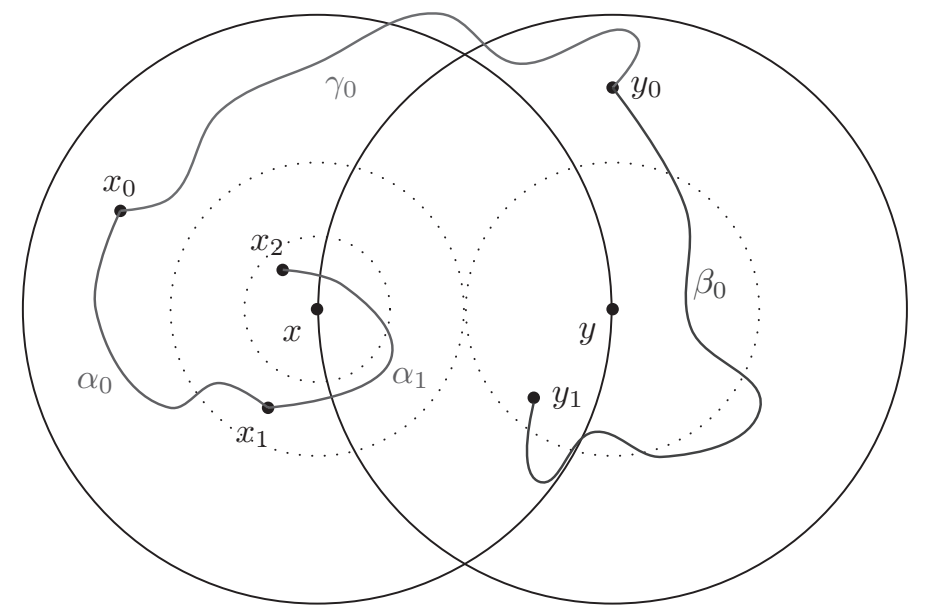

Figure 4. Constructing a uniform path

Since $x_{n} \rightarrow x$ and $y_{n} \rightarrow y$,

$$
\Gamma:=\{x\} \cup \bigcup_{0}^{\infty}\left|\alpha_{n}\right| \cup\left|\gamma_{0}\right| \cup \bigcup_{0}^{\infty}\left|\beta_{n}\right| \cup\{y\}
$$

is the trajectory of a path $\gamma$ in $U$ that joins $x$ and $y$. As

$$
\ell\left(\alpha_{n}\right) \leq b\left|x_{n}-x_{n+1}\right| \leq 3 b R / 2^{n+1}, \quad \ell\left(\beta_{n}\right) \leq 3 b R / 2^{n+1},
$$

and $\ell\left(\gamma_{0}\right) \leq b\left|x_{0}-y_{0}\right| \leq 3 b R$, we find that

$$
\ell(\gamma) \leq 3 b R+3 b R \sum_{0}^{\infty} \frac{1}{2^{n}}=9 b R=9 b|x-y| .
$$

It remains to demonstrate that $\gamma$ is a double $a$-cone path.

Let $z \in|\gamma|$. We examine the cases $z \in\left|\gamma_{0}\right|$ or $z \in\left|\alpha_{n}\right|$ for some $n$, the case $z \in\left|\beta_{n}\right|$ being similar to the latter of these. In each case: when $z$ is close to an endpoint, the double cone condition holds because the endpoints are far 
from the boundary, and when $z$ is in the middle of the path, the double cone condition holds because $z$ is far from the endpoints.

Suppose $z \in\left|\gamma_{0}\right|$. If $\left|z-x_{0}\right| \leq R / 2 c$, then

$$
d(z) \geq d\left(x_{0}\right)-\frac{R}{2 c} \geq \frac{R}{2 c}=\frac{9 b R}{18 b c} \geq \frac{\ell(\gamma)}{18 b c} .
$$

A similar argument gives $18 b c d(z) \geq \ell(\gamma)$ when $\left|z-y_{0}\right| \leq R / 2 c$. Assume that $\left|z-x_{0}\right| \wedge\left|z-y_{0}\right| \geq R / 2 c$. Since $\gamma_{0}$ is a double $b$-cone path,

$$
\begin{aligned}
b d(z) & \geq \ell\left(\gamma_{0}\left[z, x_{0}\right]\right) \wedge \ell\left(\gamma_{0}\left[z, y_{0}\right]\right) \geq \frac{R}{2 c}=\frac{9 b R}{18 b c} \\
& \geq \frac{1}{18 b c}[\ell(\gamma[z, x]) \wedge \ell(\gamma[z, y])] .
\end{aligned}
$$

Suppose $z \in\left|\alpha_{n}\right|$. Note that

$$
\ell(\gamma[z, x]) \leq \sum_{k=n}^{\infty} \ell\left(\alpha_{k}\right) \leq 3 b R \sum_{k=n}^{\infty} \frac{1}{2^{k+1}}=\frac{3 b R}{2^{n}} .
$$

Recall that $d\left(x_{k}\right) \geq R_{k} / c=R /\left(2^{k} c\right)$. If $\left|z-x_{n}\right| \wedge\left|z-x_{n+1}\right| \leq R_{n+1} / 2 c$, we deduce that

$$
d(z) \geq \frac{R_{n+1}}{2 c}=\frac{R}{2^{n+2} c}=\frac{3 b R}{12 b c 2^{n}} \geq \frac{\ell(\gamma[z, x])}{12 b c} .
$$

Finally, assume $\left|z-x_{n}\right| \wedge\left|z-x_{n+1}\right| \geq R_{n+1} / 2 c$. Since $\alpha_{n}$ is a double $b$-cone path,

$$
b d(z) \geq \ell\left(\alpha_{n}\left[z, x_{n}\right]\right) \wedge \ell\left(\alpha_{n}\left[z, x_{n+1}\right]\right) \geq \frac{R_{n+1}}{2 c}=\frac{3 b R}{12 b c 2^{n}} \geq \frac{\ell(\gamma[z, x])}{12 b c} .
$$

The interested reader is invited to furnish a proof of the converse.

\subsection{Proof of Proposition $B$}

This is a direct consequence of the following more precise result. Note that the sufficiency does not require a length space setting, however, the necessity does. This result is similar to [11, Lemmas 9.15, 9.16].

Proposition 4.2. Let $X$ be a complete doubling metric space, $U \subset X$ be an open subspace with $\operatorname{bd}(U) \neq \emptyset$, and put $A:=X \backslash U$. Suppose that for each $\left(X_{\infty}, A_{\infty} ; a_{\infty}\right)$ in $\operatorname{Tan}_{\mathrm{b}}(X, A), a_{\infty} \in \operatorname{bd}\left(A_{\infty}\right)$. Then $U$ is plump in $X$. Conversely, if $X$ is a length space and $U$ is c-plump, then for each $\left(X_{\infty}, A_{\infty} ; a_{\infty}\right)$ in $\operatorname{Tan}_{\mathrm{b}}(X, A), a_{\infty} \in \operatorname{bd}\left(A_{\infty}\right)$ and moreover $U_{\infty}:=X_{\infty} \backslash A_{\infty}$ is also c-plump. Neither of the latter implications may hold if $X$ is not a length space. 
Proof of Sufficiency. Suppose $U$ fails to be plump. Then, according to Remark 2.7(c), for each $n \in \mathrm{N}$ we can find a point $a_{n} \in \operatorname{bd}(U)=\operatorname{bd}(A)$ and a $\tau_{n} \in(0, \operatorname{diam}(\operatorname{bd}(U)))$ such that for all $x \in U \cap \overline{\mathrm{B}}\left(a_{n} ; \tau_{n}\right), d(x):=$ $\operatorname{dist}(x, \operatorname{bd}(U))<\tau_{n} / n$. As discussed above, we obtain (by taking appropriate subsequences as necessary) $\left(X_{\infty}, A_{\infty} ; a_{\infty}\right) \in \operatorname{Tan}_{\mathrm{b}}(X, A)$ with $\left(X_{\infty} ; a_{\infty}\right)$ and $\left(A_{\infty} ; a_{\infty}\right)$ the pointed Gromov-Hausdorff limits of $\left(X_{n} ; a_{n}\right)$ and $\left(A_{n} ; a_{n}\right)$ respectively.

We claim that $\mathrm{B}_{\infty}\left(a_{\infty} ; 1\right) \cap X_{\infty} \subset A_{\infty}$, which of course means that $a_{\infty} \in$ $\operatorname{int}\left(A_{\infty}\right)$. To see this, let $z_{\infty} \in \mathrm{B}_{\infty}\left(a_{\infty} ; 1\right) \cap X_{\infty}$. Then there is a sequence $\left(z_{n}\right)_{1}^{\infty}$ in $Y$ with $z_{n} \in X_{n}$ for all $n \in \mathrm{N}$ and $d_{\infty}\left(z_{n}, z_{\infty}\right) \rightarrow 0$. If infinitely many $z_{n}$ belong to $A$, then by Fact 3.1 we obtain $z_{\infty} \in A_{\infty}$. Thus we assume $z_{n} \in U$ for all $n$, and also that $z_{n} \in \overline{\mathrm{B}}_{n}\left(a_{n} ; 1\right):=\overline{\mathrm{B}}_{\infty}\left(a_{n} ; 1\right) \cap X_{n}$. Then $1 \geq d_{n}\left(z_{n}, a_{n}\right)=\left|z_{n}-a_{n}\right| / \tau_{n}$, so $z_{n} \in \overline{\mathrm{B}}\left(a_{n} ; \tau_{n}\right)$. Therefore, by our choices of $a_{n}$ and $\tau_{n}, d\left(z_{n}\right)=\operatorname{dist}\left(z_{n} \operatorname{bd}(U)\right) \leq \tau_{n} / n$.

Since $\operatorname{bd}(U)$ is closed and $X$ is proper, there are points $y_{n} \in \operatorname{bd}(U) \subset A$ with $\left|z_{n}-y_{n}\right|=d\left(z_{n}\right) \leq \tau_{n} / n$, so $d_{\infty}\left(z_{n}, y_{n}\right) \leq 1 / n$. Thus $d_{\infty}\left(y_{n}, z_{\infty}\right) \rightarrow 0$. Again by Fact 3.1, it now follows that $z_{\infty} \in A_{\infty}$.

Proof of Necessity. Suppose $X$ is a complete doubling length metric space and $U$ is $c$-plump. (Then $X$ is proper and hence geodesic, and so is every $X_{\infty}$.) Note that in this setting, $d(z)=\operatorname{dist}(z, \operatorname{bd}(U))=\operatorname{dist}(z, A)$.

Let $\left(X_{\infty}, A_{\infty} ; a_{\infty}\right) \in \operatorname{Tan}_{\mathrm{b}}(X, A)$ be given. First we prove that $a_{\infty} \in$ $\operatorname{bd}\left(A_{\infty}\right)$. Note that each $U_{n}:=X_{n} \backslash A_{n}$ is also $c$-plump. We may - and do - assume that $\operatorname{diam}\left(X_{n}\right) \rightarrow \infty$. (This is clear if $X$ is unbounded. Suppose $X$ is bounded. Then $X$, being proper, is compact, so by passing to subsequences we may assume that $a_{n} \rightarrow a \in \operatorname{bd}(A)$ and $\tau_{n} \rightarrow \tau \geq 0$. If $\tau=0$, then $\operatorname{diam}\left(X_{n}\right)=\operatorname{diam}(X) / \tau_{n} \rightarrow \infty$; assume $\tau>0$. Then $\left(X_{\infty}, A_{\infty} ; a_{\infty}\right)$ is isometric to $\left(\tau^{-1} X, \tau^{-1} A ; a\right)$ and since $a \in \operatorname{bd}(A)=\operatorname{bd}\left(\tau^{-1} A\right), a_{\infty} \in$ $\operatorname{bd}\left(A_{\infty}\right)$.)

We demonstrate that each open neighborhood of $a_{\infty}$ contains points of $U_{\infty}:=X_{\infty} \backslash A_{\infty}$. To this end, let $\varepsilon>0$ be given. We may assume that for all $n \in \mathrm{N}, r:=\varepsilon / 2 \in \Delta\left(X_{n}\right)$. The plumpness hypothesis guarantees that there exist points $z_{n} \in \overline{\mathrm{B}}_{n}\left(a_{n} ; r\right):=\overline{\mathrm{B}}_{\infty}\left(a_{n} ; r\right) \cap X_{n}$ with $\operatorname{dist}_{\infty}\left(z_{n}, A_{n}\right) \geq r / c$.

Since $d_{\infty}\left(a_{n}, a_{\infty}\right) \rightarrow 0$, the tail of the sequence $\left(z_{n}\right)_{1}^{\infty}$ lies in the compact ball $\overline{\mathrm{B}}_{\infty}\left(a_{\infty} ; 2 r\right) \subset \bar{Y}$. (Indeed, for all sufficiently large $n, d_{\infty}\left(z_{n}, a_{\infty}\right) \leq$ $d_{\infty}\left(z_{n}, a_{n}\right)+d_{\infty}\left(a_{n}, a_{\infty}\right)<2 r$. $)$ Thus $\left(z_{n}\right)_{1}^{\infty}$ subconverges to some point $z_{\infty} \in \bar{Y}$. According to Fact $3.1, z_{\infty} \in X_{\infty}$.

Since $d_{\infty}\left(z_{n}, a_{n}\right) \leq r$ for all $n, d_{\infty}\left(z_{\infty}, a_{\infty}\right) \leq r$, so $z_{\infty} \in \overline{\mathrm{B}}_{\infty}\left(a_{\infty} ; r\right) \subset$ $\mathrm{B}_{\infty}\left(a_{\infty} ; \varepsilon\right)$. Also, since $\operatorname{dist}_{\infty}\left(z_{n}, A_{n}\right) \geq r / c$ for all $n$, $\operatorname{dist}_{\infty}\left(z_{\infty}, A_{\infty}\right) \geq$ $r / c>0$, so $z_{\infty} \notin A_{\infty}$.

Now we verify that $U_{\infty}$ is $c$-plump. To this end, fix a point $x_{\infty} \in U_{\infty}$ and 
let $r \in\left(0, \operatorname{diam}_{\infty}\left(U_{\infty}\right)\right)$. We may assume $r_{n}:=\tau_{n} r<\operatorname{diam}(U)$. (To see this, approximate points in $U_{\infty}$ that have distance larger than $r$.) Also, there is a sequence $\left(x_{n}\right)_{1}^{\infty} \subset \bigsqcup_{1}^{\infty} X_{n}$ with $d_{\infty}\left(x_{n}, x_{\infty}\right) \rightarrow 0$ and we may assume that for all $n, x_{n} \in U$.

Since $U$ is $c$-plump, there are $z_{n} \in U \cap \overline{\mathrm{B}}\left(x_{n} ; r_{n}\right)$ with $d\left(z_{n}\right)=\operatorname{dist}\left(z_{n}, A\right) \geq$ $r_{n} / c$. Since $\bar{Y}$ is proper, by passing to a subsequence we may assume that $d_{\infty}\left(z_{n}, z_{\infty}\right) \rightarrow 0$ for some $z_{\infty} \in Y$; by Fact 3.1, $z_{\infty} \in X_{\infty}$. Then $z_{\infty} \in$ $\overline{\mathrm{B}}_{\infty}\left(x_{\infty} ; r\right)$ and, since $\operatorname{dist}_{\infty}\left(z_{\infty}, A_{\infty}\right) \geq r / c$, we also have $z_{\infty} \in U_{\infty}$.

Finally, we illustrate why the length space hypothesis is needed in the above. In both examples below we could arrange for $X$ to be path connected; we leave confirmation of this detail for the interested reader (but note that the 'floating pieces' can be joined to the horizontal line $y=1$ by using appropriate vertical segments).

For our first example, note that $U:=\bigcup_{n=0}^{\infty}\left(2^{n}, 2^{n+1}\right) \times\{0\}$ is a plump open subspace of

$$
X:=(\mathrm{R} \times\{0\}) \cup \bigcup_{n=1}^{\infty}\left(\left[2^{n}-1,2^{n}+1\right] \times\left\{1 / n^{2}\right\}\right) \subset \mathrm{R}^{2} ;
$$

here we use the restriction of Euclidean distance in $R^{2}$. See Figure 5.

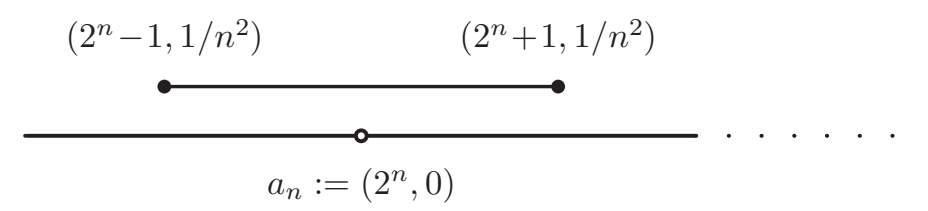

FIGURE 5. $U:=\bigcup_{n=0}^{\infty}\left(2^{n}, 2^{n+1}\right) \times\{0\} \subset X \subset \mathrm{R}^{2}$

Taking base-points $a_{n}:=\left(2^{n}, 0\right)$ and scaling with $\tau_{n}^{-1}=n$, we find that $\left(X_{n} ; a_{n}\right)$ and $\left(A_{n} ; a_{n}\right):=\left(\tau_{n}^{-1}(X \backslash U) ; a_{n}\right)$ both have pointed Gromov-Hausdorff limit $(R ; 0)$, so the limit base-point 0 is an interior point and not a boundary point.

For our second example (see Figure 6), we start with the point set

$$
B:=\{0\} \cup\left\{\frac{1}{k+1}+\frac{j}{k^{2}(k+1)}: j \in[0, k) \cap \mathrm{Z}, k \in \mathrm{N}\right\} \subset[0,1 / 2]
$$

that is formed by inserting $k-1$ equally spaced points between $1 /(k+1)$ and $1 / k$ thereby producing $k$ equally spaced gaps in $[1 /(k+1), 1 / k]$. It is readily observed that $\mathrm{R} \backslash B$ is not plump. Next, for each $n \in \mathrm{N}$ set $B_{n}:=n^{-1} B+2^{n}$. Again, consider $U:=\bigcup_{n=0}^{\infty}\left(2^{n}, 2^{n+1}\right) \times\{0\}$ which is now a plump open 


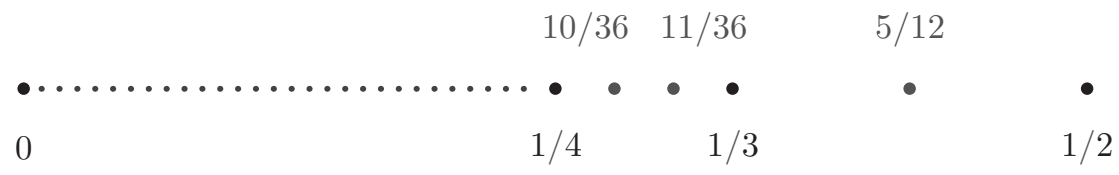

FIGURE 6. The points $\frac{k^{2}+j}{k^{2}(k+1)}$ in $B$ for $0 \leq j<k \in\{1,2,3\}$

subspace of

$$
X:=(\mathrm{R} \times\{0\}) \cup \bigcup_{n=1}^{\infty}\left(B_{n} \times\left\{1 / n^{2}\right\}\right) \subset \mathrm{R}^{2}
$$

again we use the restriction of Euclidean distance. Taking base-points $a_{n}:=$ $\left(2^{n}, 0\right)$ and scaling with $\tau_{n}^{-1}=n$, we find that $\left(X_{n} ; a_{n}\right)$ and $\left(A_{n} ; a_{n}\right):=$ $\left(\tau_{n}^{-1}(X \backslash U) ; a_{n}\right)$ have pointed Gromov-Hausdorff limits $(\mathrm{R} ; 0)$ and $(B ; 0)$ respectively, and so $U_{\infty}:=X_{\infty} \backslash A_{\infty}=\mathrm{R} \backslash B$ which is not plump.

\subsection{Proof of Theorem $A$}

Let $X$ be a $b$-annular quasiconvex complete doubling metric space. According to Fact 2.2(a), $X$ is quasiconvex, so it is bilipschitz equivalent to a length space, and therefore - since $X$ is proper - it is bilipschitz equivalent to a geodesic space. Employing Fact 3.2 to obtain bilipschitz maps at the tangent space level, and noting that our hypotheses and conclusions are bilipschitz quasi-invariant, it now follows that we may assume that $X$ is a $b$-annular quasiconvex complete doubling geodesic space.

Everywhere below, $U$ is an open connected subspace of $X$ with $\operatorname{bd}(U) \neq$ $\emptyset$ and $A:=X \backslash U$. As already mentioned, we obtain stronger necessary conditions than those stated in the Introduction.

Proof of Necessity. We assume $U$ is $c$-uniform; here we only require that $X$ be a complete doubling length space. According to Proposition $\mathrm{C}, U$ is plump in $X$. Suppose we are given $\left(X_{\infty}, A_{\infty} ; a_{\infty}\right) \in \operatorname{Tan}_{\mathrm{b}}(X, A)$. Since $U$ is plump, Proposition 4.2 tells us that $a_{\infty} \in \mathrm{bd}\left(A_{\infty}\right)$. It remains to demonstrate that $U_{\infty}:=X_{\infty} \backslash A_{\infty}$ is connected. In fact we show that $U_{\infty}$ is $c$-uniform.

To this end, let $x_{\infty}, y_{\infty} \in U_{\infty}$ and select sequences $\left(x_{n}\right)_{1}^{\infty},\left(y_{n}\right)_{1}^{\infty} \subset \bigsqcup_{1}^{\infty} X_{n}$ that converge in $\bar{Y}$ to $x_{\infty}, y_{\infty}$ respectively. We may assume that for all $n$ both $x_{n}, y_{n} \in U$. Since $U$ is $c$-uniform, there is a $c$-uniform path $\gamma_{n}$ in $U$ joining $x_{n}, y_{n}$. See Figure 2, but ignore the annulus!

The Arzela-Ascoli theorem provides a subsequence of $\left(\gamma_{n}\right)_{1}^{\infty}$ that converges uniformly to a rectifiable path $\gamma_{\infty}$ in $X_{\infty}$ joining $x_{\infty}$ to $y_{\infty}$. To check this, put $r:=d_{\infty}\left(x_{\infty}, a_{\infty}\right) \vee d_{\infty}\left(y_{\infty}, a_{\infty}\right)$ and $R:=(c+1)(2 r+1)$. Then for all 
sufficiently large $n \in \mathrm{N}$ and all $z \in\left|\gamma_{n}\right|$,

$$
d_{\infty}\left(z, x_{\infty}\right) \vee d_{\infty}\left(z, y_{\infty}\right) \leq c(2 r+1) \quad \text { so } \quad d_{\infty}\left(z, a_{\infty}\right) \leq R,
$$

and therefore $\left|\gamma_{n}\right| \subset \overline{\mathrm{B}}_{\infty}\left(a_{\infty} ; R\right)$. Assuming each $\gamma_{n}$ is parameterized with respect to arclength, we have $\left(\gamma_{n}\right)_{1}^{\infty}$ equicontinuous and thus uniformly subconvergent; e.g., see [8, 2.3(iv), p. 35; 2.5.14, p. 47] or [6, 1.23, p. 14; 3.10, p. 36].

Now, after selecting a uniformly convergent subsequence, and relabeling, we obtain

$$
\ell_{\infty}\left(\gamma_{\infty}\right)=\lim _{n \rightarrow \infty} \ell_{\infty}\left(\gamma_{n}\right) \leq c \lim _{n \rightarrow \infty} d_{\infty}\left(x_{n}, y_{n}\right)=c d_{\infty}\left(x_{\infty}, y_{\infty}\right),
$$

so $\gamma_{\infty}$ is a $c$-quasiconvex path joining $x_{\infty}, y_{\infty}$. Moreover,

$$
\begin{aligned}
\forall z_{\infty} & :=\gamma_{\infty}(t)=\lim _{n \rightarrow \infty} \gamma_{n}(t)=\lim _{n \rightarrow \infty} z_{n} \quad\left(\text { where } z_{n}:=\gamma_{n}(t)\right), \\
d_{\infty}\left(z_{\infty}\right) & =\operatorname{dist}_{\infty}\left(z_{\infty}, A_{\infty}\right)=\lim _{n \rightarrow \infty} \operatorname{dist}_{\infty}\left(z_{n}, A_{\infty}\right)=\lim _{n \rightarrow \infty} d_{\infty}\left(z_{n}\right) \\
& \geq c^{-1} \lim _{n \rightarrow \infty}\left[\ell_{\infty}\left(\gamma_{n}\left[x_{n}, z_{n}\right]\right) \wedge \ell_{\infty}\left(\gamma_{n}\left[y_{n}, z_{n}\right]\right)\right] \\
& \geq c^{-1}\left[\ell_{\infty}\left(\gamma_{\infty}\left[x_{\infty}, z_{\infty}\right]\right) \wedge \ell_{\infty}\left(\gamma_{\infty}\left[y_{\infty}, z_{\infty}\right]\right)\right] .
\end{aligned}
$$

Thus $\gamma_{\infty}$ is a double $c$-cone path in $U_{\infty}$.

Proof OF Sufficiency. We establish the contrapositive. Thanks to Propositions $\mathrm{B}$ and $\mathrm{C}$, we may assume that $U$ is a non-uniform plump subspace of $X$. Our goal is to exhibit a tangent subspace triple $\left(X_{\infty}, A_{\infty} ; a_{\infty}\right)$ in $\operatorname{Tan}_{\mathrm{b}}(X, A)$ with $X_{\infty} \backslash A_{\infty}$ non-connected. The argument here is similar to, but more technical than, the proof of Theorem D.

Fix $c \geq b$ so that $U$ is $c$-plump. Since $U$ is non-uniform, an appeal to Proposition C provides, for each $n \in \mathrm{N}, 3 c$-proximate points $x_{n}, y_{n}$ with the property that

$$
x_{n} \text { and } y_{n} \text { cannot be joined by an } n \text {-uniform path in } U \text {. }
$$

We assume that $r_{n}:=d\left(x_{n}\right) \leq d\left(y_{n}\right)$. According to Lemma 2.4, $y_{n} \notin \overline{\mathrm{B}}\left(x_{n}\right)$. Since $x_{n}, y_{n}$ are $3 c$-proximate,

$$
(4 c)^{-1} \leq d\left(x_{n}\right) / d\left(y_{n}\right) \leq 4 c \quad \text { and } \quad r_{n}<\left|x_{n}-y_{n}\right| \leq 3 c d\left(y_{n}\right) \leq 12 c^{2} r_{n} .
$$

Select points $a_{n} \in \operatorname{bd}(U)=\operatorname{bd}(A)$ with $\left|x_{n}-a_{n}\right|=d\left(x_{n}\right)=r_{n}$. Put $B_{n}:=$ $A \cap\left[36 c^{2} \overline{\mathrm{B}}\left(x_{n}\right)\right]$. If $B_{n} \subset \overline{\mathrm{B}}\left(a_{n} ; r_{n} / 2 b\right)$, then we could employ Lemma 2.5 to exhibit a $13 c^{2}$-uniform path joining $x_{n}, y_{n}$ in $U$ and this would contradict 


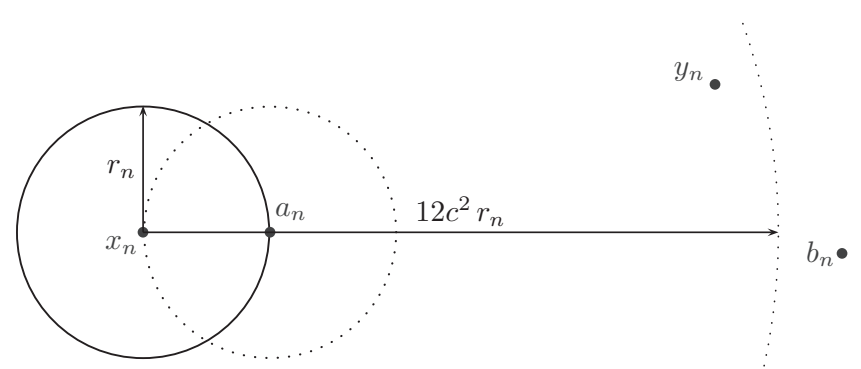

FIGURE 7. The points $x_{n}, y_{n}, a_{n}, b_{n}$

(4.3) for large $n$. Thus we can - and do - assume that there exists a point $b_{n} \in B_{n} \backslash \mathrm{B}\left(a_{n} ; r_{n} / 2 b\right)$. See Figure 7.

We now have $x_{n}, y_{n} \in U, a_{n} \in \operatorname{bd}(A)$ and $b_{n} \in A$ with $\left|x_{n}-a_{n}\right|=d\left(x_{n}\right)=$ $r_{n}$ and

$$
r_{n} \leq\left|x_{n}-y_{n}\right| \leq 12 c^{2} r_{n} \quad \text { and } \quad r_{n} / 2 b \leq \tau_{n}:=\left|a_{n}-b_{n}\right| \leq 37 c^{2} r_{n} .
$$

Using the base-points $a_{n}$ and scales $\tau_{n}^{-1}$ we construct a tangent subspace triple $\left(X_{\infty}, A_{\infty}, a_{\infty}\right)$ in $\operatorname{Tan}_{\mathrm{b}}(X, A)$. We note that $X_{\infty}$ is a complete doubling geodesic space and $A_{\infty}$ is a closed subspace, so $U_{\infty}:=X_{\infty} \backslash A_{\infty}$ is open.

We verify that $U_{\infty}$ is not connected. To this end, we assume otherwise and derive a contradiction to (4.3). Assume $U_{\infty}$ is connected. We construct paths joining $x_{n}, y_{n}$ that, for sufficiently large $n$, are $n$-uniform. Note that $U_{\infty}$, being an open connected subspace of a geodesic space, is piecewise-geodesically connected.

The sequences $\left(x_{n}\right)_{1}^{\infty},\left(y_{n}\right)_{1}^{\infty} \subset \bigsqcup_{1}^{\infty} X_{n}$ determine distinct points $x_{\infty}, y_{\infty} \in$ $U_{\infty}$. Indeed,

$$
d_{\infty}\left(x_{n}, a_{n}\right)=d_{n}\left(x_{n}, a_{n}\right)=\tau_{n}^{-1}\left|x_{n}-a_{n}\right|=r_{n} / \tau_{n} \leq 2 b
$$

which implies that

$$
\forall \text { large } n, x_{n} \in \mathrm{B}_{\infty}\left(a_{\infty} ; 3 b\right)
$$

and similarly for $\left(y_{n}\right)_{1}^{\infty}$, so by passing to subsequences we may assume that $\left(x_{n}\right),\left(y_{n}\right)$ converge to points $x_{\infty}, y_{\infty} \in Y$; then by Fact 3.1, $x_{\infty}, y_{\infty} \in X_{\infty}$. Also,

$$
d_{n}\left(x_{n}, y_{n}\right)=\tau_{n}^{-1}\left|x_{n}-y_{n}\right| \geq r_{n} / \tau_{n} \geq 1 / 37 c^{2} \Rightarrow d_{\infty}\left(x_{\infty}, y_{\infty}\right)>0,
$$


and

$$
d_{n}\left(y_{n}\right) \geq d_{n}\left(x_{n}\right)=r_{n} / \tau_{n} \Rightarrow d_{\infty}\left(y_{\infty}\right) \geq d_{\infty}\left(x_{\infty}\right):=\operatorname{dist}_{\infty}\left(x_{\infty}, A_{\infty}\right)>0
$$

so $x_{\infty}, y_{\infty} \notin A_{\infty}$. Thus $x_{\infty}, y_{\infty} \in U_{\infty}$.

Since $U_{\infty}$ is piecewise-geodesically connected, there exist $z_{0}:=x_{\infty}, z_{1}$, $\ldots, z_{m}:=y_{\infty}$ in $U_{\infty}$ such that the piecewise geodesic path

$$
\gamma_{\infty}:=\left[z_{0}, z_{1}\right] \star\left[z_{1}, z_{2}\right] \star \ldots \star\left[z_{m-1}, z_{m}\right]
$$

joins $x_{\infty}, y_{\infty}$ in $U_{\infty}$. (See Figure 3, but ignore the annulus:-) Put

$$
\begin{gathered}
\delta:=\operatorname{dist}_{\infty}\left(\left|\gamma_{\infty}\right|, A_{\infty}\right), \quad \lambda:=\ell_{\infty}\left(\gamma_{\infty}\right), \quad d:=d_{\infty}\left(x_{\infty}, y_{\infty}\right), \\
C:=\frac{\lambda}{d \wedge \delta}=\frac{\lambda}{d} \vee \frac{\lambda}{\delta} \quad \text { and } \quad t:=\frac{1}{10}\left(\delta \wedge d \wedge \frac{1}{d}\right)
\end{gathered}
$$

Note that $\gamma_{\infty}$ is a $C$-uniform path.

If necessary, we may insert additional points $z_{i}$ (this does not change the values of any of $\delta, \lambda, d$ ) to ensure that both

$$
m \geq 10 \vee C \vee b \quad \text { and } \quad \forall 1 \leq i \leq m, \quad d_{\infty}\left(z_{i}, z_{i-1}\right)<t / 10 .
$$

An easy calculation confirms that

$$
2[(t / 5)+C d+2 b]+(t / 10 m)<10 m / t .
$$

Fix $n \in \mathrm{N}$ sufficiently large so that $n \geq 2 C$ and

$$
\begin{gathered}
d_{\infty}\left(x_{n}, x_{\infty}\right) \wedge d_{\infty}\left(y_{n}, y_{\infty}\right) \wedge d_{\infty}\left(a_{n}, a_{\infty}\right)<t / 10 m \\
\operatorname{dist}_{G_{H_{*}}}\left(\left(X_{n} ; a_{n}\right),\left(X_{\infty} ; a_{\infty}\right)\right)<t / 10 m \text { and } \\
\operatorname{dist}_{\mathscr{G}_{*}}\left(\left(A_{n} ; a_{n}\right),\left(A_{\infty} ; a_{\infty}\right)\right)<t / 10 m
\end{gathered}
$$

Since

$$
d_{\infty}\left(z_{i}, a_{\infty}\right) \leq d_{\infty}\left(z_{i}, x_{\infty}\right)+d_{\infty}\left(x_{\infty}, a_{\infty}\right) \leq \lambda+2 b \leq C d+2 b<10 m / t,
$$

there are points $z_{n i} \in X_{n}$ with $d_{\infty}\left(z_{n i}, z_{i}\right)<t / 10 m$. We take $z_{n 0}:=x_{n}, z_{n m}:=$ $y_{n}$. Note that

$$
\begin{aligned}
d_{\infty}\left(z_{n i}, z_{n, i-1}\right) & \leq d_{\infty}\left(z_{n i}, z_{i}\right)+d_{\infty}\left(z_{i}, z_{i-1}\right)+d_{\infty}\left(z_{i-1}, z_{n, i-1}\right) \\
& \leq \frac{t}{10 m}+\frac{t}{10}+\frac{t}{10 m}
\end{aligned}
$$


We claim that for all $1 \leq i \leq m,\left[z_{n i}, z_{n, i-1}\right] \subset U$ (when $z_{i n}$ are viewed as points in $X$ ) and thus

$$
\gamma_{n}:=\left[z_{n 0}, z_{n 1}\right] \star\left[z_{n 1}, z_{n 2}\right] \star \cdots \star\left[z_{n, m-1}, z_{n m}\right]
$$

is a path joining $x_{n}, y_{n}$ in $U$. In fact, we claim that

$$
\forall z \in\left|\gamma_{n}\right|, \quad d_{n}(z):=\operatorname{dist}_{n}\left(z, A_{n}\right) \geq 9 \delta / 10 .
$$

To check this claim, we first compute

$$
\begin{aligned}
\ell_{n}\left(\gamma_{n}\right) & =\sum_{i=1}^{m} d_{n}\left(z_{n i}, z_{n, i-1}\right) \leq \sum_{i=1}^{m}\left(\frac{t}{5 m}+d_{\infty}\left(z_{i}, z_{i-1}\right)\right) \\
& =\frac{t}{5}+\lambda \leq \frac{t}{5}+C d .
\end{aligned}
$$

Next, fix a point $z \in\left[z_{i n}, z_{i-1, n}\right]$. Then

$$
d_{\infty}\left(z_{i n}, z\right) \leq d_{\infty}\left(z_{i n}, z_{i-1, n}\right) \leq \frac{t}{10}+\frac{t}{5 m} .
$$

Now pick $w \in A_{n}$ with $d_{n}(z, w)=\operatorname{dist}_{n}\left(z, A_{n}\right)$ (so $|z-w|=d(z)$ and possibly $w=z$ ). Then

$$
\begin{aligned}
d_{\infty}\left(w, a_{\infty}\right) & \leq d_{\infty}(w, z)+d_{\infty}\left(z, a_{\infty}\right) \leq 2 d_{\infty}\left(z, a_{n}\right)+d_{\infty}\left(a_{n}, a_{\infty}\right) \\
& \leq 2\left[d_{\infty}\left(z, x_{n}\right)+d_{\infty}\left(x_{n}, a_{n}\right)\right]+d_{\infty}\left(a_{n}, a_{\infty}\right) \\
& \leq 2\left[\ell_{n}\left(\gamma_{n}\right)+2 b\right]+\frac{t}{10 m} \\
& \leq 2\left(\frac{t}{5}+C d+2 b\right)+\frac{t}{10 m} \leq \frac{10 m}{t}
\end{aligned}
$$

here the last inequality follows from (4.4). Thus there is a point $w_{\infty} \in A_{\infty}$ such that $d_{\infty}\left(w, w_{\infty}\right)<t / 10 m$. Since $w_{\infty} \in A_{\infty}$ and $z_{i} \in\left|\gamma_{\infty}\right|$,

$$
\begin{aligned}
\delta & =\operatorname{dist}_{\infty}\left(\left|\gamma_{\infty}\right|, A_{\infty}\right) \leq d_{\infty}\left(z_{i}, w_{\infty}\right) \\
& \leq d_{\infty}\left(z_{i}, z_{n i}\right)+d_{\infty}\left(z_{n i}, z\right)+d_{\infty}(z, w)+d_{\infty}\left(w, w_{\infty}\right) \\
& \leq \frac{t}{10 m}+\left(\frac{t}{10}+\frac{t}{5 m}\right)+d_{\infty}(z, w)+\frac{t}{10 m} \\
& =\frac{m+4}{10 m} t+d_{\infty}(z, w) \leq \frac{t}{5}+d_{\infty}(z, w),
\end{aligned}
$$

and therefore $d_{n}(z)=d_{\infty}(z, w) \geq \delta-t / 5 \geq 9 \delta / 10$ establishing (4.5). 
Finally, we demonstrate that $\gamma_{n}$ is a $2 C$-uniform path joining $x_{n}, y_{n}$ in $U$. Since $n \geq 2 C$, this directly contradicts (4.3). We begin by noting that

$$
\begin{aligned}
\left|d-d_{\infty}\left(x_{n}, y_{n}\right)\right| & =\left|d_{\infty}\left(x_{\infty}, y_{\infty}\right)-d_{\infty}\left(x_{n}, y_{n}\right)\right| \\
& \leq d_{\infty}\left(x_{\infty}, x_{n}\right)+d_{\infty}\left(y_{\infty}, y_{n}\right) \leq \frac{t}{5 m},
\end{aligned}
$$

so $d_{\infty}\left(x_{n}, y_{n}\right) \geq d-t / 5 m \geq 10 t-t / 5 m \geq 9 t$ and also

$$
\frac{t}{5}\left(1+\frac{C}{m}\right) \leq \frac{2 t}{5}<d_{\infty}\left(x_{n}, y_{n}\right) .
$$

Thus from (4.6) we obtain

$$
\begin{aligned}
\ell_{n}\left(\gamma_{n}\right) & \leq \frac{t}{5}+\lambda \leq \frac{t}{5}+C d \leq \frac{t}{5}+C\left(d_{\infty}\left(x_{n}, y_{n}\right)+\frac{t}{5 m}\right) \\
& =\frac{t}{5}\left(1+\frac{C}{m}\right)+C d_{\infty}\left(x_{n}, y_{n}\right) \leq(C+1) d_{\infty}\left(x_{n}, y_{n}\right)
\end{aligned}
$$

and we see that $\gamma_{n}$ is a $2 C$-quasiconvex path. Recalling (4.5), and using (4.6) again, for each $z \in\left|\gamma_{n}\right|$ we have

$$
\begin{aligned}
\ell_{n}\left(\gamma_{n}\left[x_{n}, z\right]\right) \wedge \ell_{n}\left(\gamma_{n}\left[y_{n}, z\right]\right) & \leq \ell_{n}\left(\gamma_{n}\right) \leq \frac{t}{5}+\lambda \leq \frac{t}{5}+C \delta \\
& \leq(C+1 / 50) \delta \leq 2 C d_{n}(z)
\end{aligned}
$$

and therefore $\gamma_{n}$ is a double $2 C$-cone path.

\section{REFERENCES}

1. Aikawa, H., Potential-theoretic characterizations of nonsmooth domains, Bull. London Math. Soc. 36 (2004), 469-482.

2. Aikawa, H., Characterization of a uniform domain by the boundary Harnack principle, pp. 117 in: Harmonic Analysis and its Applications, Yokohama Publ., Yokohama 2006.

3. Ambrosio, L., and Tilli, P., Topics on Analysis in Metric Spaces, Oxford Lecture Series Math. Appl. 25, Oxford Univ. Press, Oxford 2004.

4. Björn, J., and Shanmugalingam, N., Poincaré inequalities, uniform domains and extension properties for Newton-Sobolev functions in metric spaces, J. Math. Anal. Appl. 332 (2007), 190-208.

5. Bonk, M., Heinonen, J., and Koskela, P., Uniformizing Gromov hyperbolic spaces, Astérisque 270 (2001), 1-99.

6. Bridson, M. R., and Haefliger, A., Metric Spaces of Non-positive Curvature, Grundlehren math. Wiss. 319, Springer, Berlin 1999.

7. Buckley, S., Herron, D. A., and Xie, X., Metric space inversions, quasihyperbolic distance and uniform spaces, Indiana Univ. Math. J. 57 (2008), 837-890. 
8. Burago, D., Burago, Y., and Ivanov, S., A Course in Metric Geometry, Graduate Studies in Math. 33, Amer. Math. Soc., Providence, RI 2001.

9. Capogna, L., Garofalo, N., and Nhieu, D.-M., Examples of uniform and NTA domains in Carnot groups, pp. 103-121 in: Proceedings on Analysis and Geometry, Proc. Novosibirsk Akademgorodok 1999, Izdat. Ross. Akad. Nauk Sib. Otd. Inst. Mat., Novosibirsk 2000.

10. Capogna, L., and Tang, P., Uniform domains and quasiconformal mappings on the Heisenberg group, Manuscripta Math. 86 (1995), 267-281.

11. David, G., and Semmes, S., Fractured Fractals and Broken Dreams. Self-similar Geometry through Metric and Measure, Oxford Lecture Series Math. Appl. 7, Oxford Univ. Press, Oxford 1997.

12. Gehring, F. W., Uniform domains and the ubiquitous quasidisk, Jahresber. Deutsch. Math.Verein. 89 (1987), 88-103.

13. Greshnov, A. V., On uniform and NTA-domains on Carnot groups, Sibirsk. Mat. Zh. 42 (2001), 1018-1035; transl. in Siberian Math. J. 42 (2001), 851-864.

14. Gromov, M., Groups of polynomial growth and expanding maps, Inst. Hautes Études Sci. Publ. Math. 53 (1981), 53-73.

15. Hakobyan, H., and Herron, D. A., Euclidean quasiconvexity, Ann. Acad. Sci. Fenn. Math. 33 (2008), 205-230.

16. Heinonen, J., and Koskela, P., Quasiconformal maps in metric spaces with controlled geometry, Acta Math. 181 (1998), 1-61.

17. Herron, D. A., Gromov-Hausdorff distance for pointed metric spaces, in preparation.

18. Herron, D. A., and Koskela, P., Uniform, Sobolev extension and quasiconformal circle domains, J. Anal. Math. 57 (1991), 172-202.

19. Herron, D. A., Shanmugalingham, N., and Xie, X., Uniformity from Gromov hyperbolicity, Illinois J. Math. 52 (2008), 1065-1109.

20. John, F., Rotation and strain, Comm. Pure Appl. Math. 14 (1961), 391-413.

21. Jones, P. W., Quasiconformal mappings and extendability of functions in Sobolev spaces, Acta Math. 147 (1981), 71-88.

22. Korte, R., Geometric implications of the Poincaré inequality, Results Math. 50 (2007), 93107.

23. Mackay, J., Spaces with conformal dimension greater than one, preprint.

24. Martio, O., Definitions for uniform domains, Ann. Acad. Sci. Fenn. Ser. A I Math. 5 (1980), 197-205.

25. Martio, O., and Sarvas, J., Injectivity theorems in plane and space, Ann. Acad. Sci. Fenn. Ser. A I Math. 4 (1978/79), 383-401.

26. Petersen, P., Gromov-Hausdorff convergence of metric spaces, pp. 489-504 in: Differential Geometry: Riemannian Geometry, Proc. Los Angeles 1990, Proc. Symp. Pure Math. 54:3, Amer. Math. Soc., Providence, RI 1993.

27. Väisälä, J., Uniform domains, Tôhoku Math. J. (2) 40 (1988), 101-118.

28. Väisälä, J., Exhaustions of John domains, Ann. Acad. Sci. Fenn. Math. 19 (1994), 47-57.

UNIVERSITY OF CINCINNATI

DEPARTMENT OF MATHEMATICAL SCIENCES

839 OLD CHEMISTRY BUILDING

PO BOX 210025

CINCINNATI, OH 45221-0025

U.S.A.

E-mail: David.Herron@math.UC.edu 